\title{
A tutorial on applications of power watershed optimization to image processing
}

\author{
Sravan Danda ${ }^{1}$, Aditya Challa ${ }^{2}$, B. S. Daya Sagar ${ }^{3}$, and Laurent Najman ${ }^{4, a}$ \\ ${ }^{1}$ Computer Science and Information Systems, APPCAIR, BITS-Pilani K K Birla Goa Campus, Sancoale, Goa, India \\ 2 Computer Science and Automation, Indian Institute of Science, Bengaluru, India \\ 3 Systems Science and Informatics Unit, Indian Statistical Institute, Bengaluru, India \\ 4 Université Gustave Eiffel, LIGM, Equipe A3SI, ESIEE, Paris, France
}

Received 6 January 2021 / Accepted 3 August 2021 / Published online 2 September 2021 (C) The Author(s), under exclusive licence to EDP Sciences, Springer-Verlag GmbH Germany, part of Springer Nature 2021

\begin{abstract}
This tutorial review paper consolidates the existing applications of the power watershed (PW) optimization framework in the context of image processing. In the literature, it is known that PW framework when applied to some well-known graph-based image segmentation and filtering algorithms such as random walker, isoperimetric partitioning, ratio-cut clustering, multi-cut and shortest path filters yield faster yet consistent solutions. In this paper, the intuition behind the working of PW framework, i.e. exploitation of contrast invariance on image data is explained. The intuitions are illustrated with toy images and experiments on simulated astronomical images. This article is primarily aimed at researchers working on image segmentation and filtering problems in application areas such as astronomy where images typically have huge number of pixels. Classic graph-based cost minimization methods provide good results on images with small number of pixels but do not scale well for images with large number of pixels. The ideas from the article can be adapted to a large class of graph-based cost minimization methods to obtain scalable segmentation and filtering algorithms.
\end{abstract}

\section{Introduction}

Machine vision applications are diverse ranging from segmentation, filtering, object detection, object localization, instance segmentation, semantic segmentation, image classification, image captioning, image reconstruction, etc. [49]. Image segmentation and filtering form the building blocks of many machine vision tasks. Historically, energy-based or cost minimizationbased approaches have been popularly used to solve image segmentation and filtering. The cost functions are designed such that the minimizers yields desired results. Also, the cost functions are constructed to incorporate an inductive bias in images namely translation invariance. In the context of image classification, translation invariance means - if object(s) in an image are vertically or horizontally translated within the image, this image is still expected to be classified as the same category (or set of categories). CNNs takes this approach to the next level, by allowing many parameters to be learned by gradient descent, with the very same cost function, and allowing the system to compute parameters that are otherwise difficult to predict or to tune. Some works additionally capitalize on

\footnotetext{
a e-mail: laurent.najman@esiee.fr (corresponding author)
}

rotation invariance by augmenting the training datasets with customized rotations. This helped to improve the performance of the cost minimization-based approaches further.

One of the less exploited aspects of image data is that of contrast invariance. Intuitively, it is clear that a human eye perceives the same set of objects in an image even if the contrast of the image is changed (see Fig. 1). In fact, the object boundaries are also expected to be intact when the contrast of the image is altered. An obvious question arises - how does one exploit the contrast-invariance nature of solutions to image processing tasks? In this article, we provide an answer to this question in the context of image segmentation and image filtering. We revisit the power watershed (PW) framework [39] and show that this optimization framework provides a formalism to impose the constraint that the solutions are contrast-agnostic. It is well known that an application of PW to classic graph-based cost minimization methods results in high-quality approximation solutions to the cost minimization problem $[1,10$ $12,17,18,21-23,54-56]$. In other words, PW exploits contrast-agnostic nature of solutions and allows one to apply classic graph-based cost minimization methods to images with very large number of pixels which were otherwise prohibited by computational constraints. In particular, astronomical images which have a huge number 

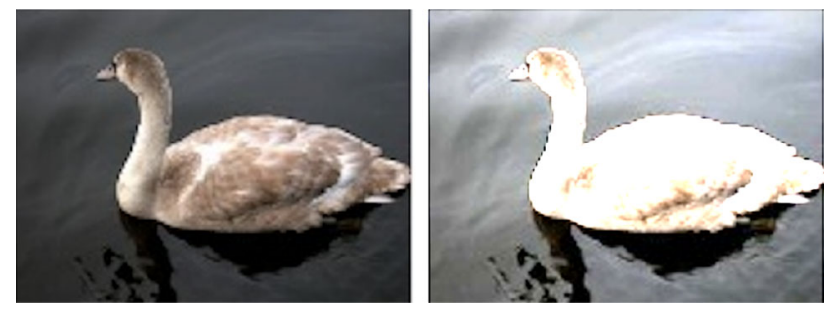

Fig. 1 Left: An image from the Weizmann 1-object database [2]. Right: Enhanced contrast of the image on left. Observe that the object boundaries are not expected to change even in the contrast enhanced image. One expects that a segmentation method applied on either of these images yields the same result

of pixels can be segmented and/or filtered with graphbased cost minimization methods using PW framework.

Recall that image segmentation and filtering form the building blocks of many image processing applications. Image segmentation and image filtering are typically used either as a pre-processing or a post-processing step depending on the machine vision task at hand. Image segmentation is an ill-posed problem. The goal of image segmentation is to cluster pixels in an image such that the clusters are 'close' to the clusters obtained by a domain expert w.r.t. some standard measures that compare closeness of clusters. Typically, each cluster obtained by a domain expert contains pixels only from one object and the number of clusters are usually much smaller when compared to the total number of pixels in the image (see Fig. 2 for a segmentation of an image obtained by a domain expert using two labels). Image filtering is another closely related ill-posed problem. Image filtering is the process of magnifying certain details while suppressing the others. Informally, image filtering can be interpreted as summarizing the content of an image by removing redundant details. For example, in Fig. 2, to identify the object boundaries, details such as feathers are irrelevant and can be ignored. Although, in this article, we deal with only image segmentation and image filtering problems specific to $2 \mathrm{D}$ images, the techniques we present are generic and can be applied to any kind of image data.

Recall that a 2D digital image consists of a finite number of pixels. Each pixel represents a small physical area and typically these pixels are square shaped. To each pixel, one associates a scalar or vector that represents the average intensity/color reflected by the corresponding area. A 2D digital image of dimensions $M \times N$ is thus a matrix of scalar/vector-valued entries with $M$ rows and $N$ columns.

$$
I:\{0,1, \ldots, M-1\} \times\{0,1, \ldots, N-1\} \rightarrow \mathcal{Z}^{\rho},
$$

where $\mathcal{Z}$ is a discrete set consisting of non-negative real numbers and and $\rho$ denotes the number of bands $(\rho=1$ in case of greyscale images and $\rho=3$ in case of color images). However, in this article, we use edge-weighted graphs to model images and work with those models instead of Eq. 1. This is because local changes such as gradients that provide information on object boundaries can be captured using the edge weights. Recall that a gradient is a dissimilarity measure between neighbouring pixels. A 2D image is represented as a 4-grid graph or a 4-adjacency graph (Von Neumann neighborhood) with vertices representing the pixels and the edge weights reflecting a similarity/dissimilarity between neighbouring pixels. Formally, if $I$ is an image of dimensions $M \times N$, we have $\mathcal{G}_{I}=(V, E, W)$ where $V$, the set of vertices represents the pixels, i.e.

$$
V=\{(i, j): 0 \leq i \leq M-1,0 \leq j \leq N-1\},
$$

the set of edges are given by

$$
\begin{aligned}
E= & \bigcup_{0 \leq i \leq M-1,0 \leq j \leq N-1} E_{i j} \\
E_{i j}= & \left\{\left\{\left(i^{\prime}, j^{\prime}\right),(i, j)\right\}:\left|i-i^{\prime}\right|+\left|j-j^{\prime}\right|=1\right. \\
& \text { and } \left.0 \leq i^{\prime} \leq M-1,0 \leq j^{\prime} \leq N-1\right\},
\end{aligned}
$$

for each $0 \leq i \leq M-1,0 \leq j \leq N-1$, and $W: E \rightarrow \mathbb{R}^{+}$ is a non-negative real valued function on the set of edges. The edge weights either represent a similarity or a dissimilarity depending on the application at hand. Typically, the edge weights are obtained using monotonic functions of a standard norm of the difference of the pixel intensities/colors of pixels incident on the edge.

Recall that the increase (respectively, decrease) in contrast of an image in the classic sense is obtained by multiplying intensity/each coordinate in the color vector of each pixel by a constant non-negative number greater (respectively, lesser) than one. For the edgeweighted graph model, this is equivalent to: the contrast of an image is increased (respectively, decreased) when the edge weights are magnified (respectively, diminished). Mathematically, a magnifying (respectively, shrinking) operation on the edge weights is a function on the edge weights with derivative greater (respectively, lesser) than one. Also, a change in contrast does not alter the relative ordering of edge weights. Mathematically, this is equivalent to restricting the operations on the edge weights to be strictly increasing functions. Thus, the answer to the question posed in the second paragraph, i.e. contrast invariance can be obtained by considering only the 'solutions that are invariant to strictly increasing functions on the edge weights of the graph constructed on the image'. Typically, a graph constructed to model an image is referred to as the image graph irrespective of whether the edge weights reflect a similarity or a dissimilarity or a combination of both. In the rest of the article, we use the term 'image graph' to refer to the 'graph constructed on an image'.

In the literature, there are plenty of graph-based methods to obtain image segmentation and filtering. For segmentation, broadly they fall under two overlapping categories namely graph-partitioning methods [3, 51] and variational cost minimization [37]. Both these 

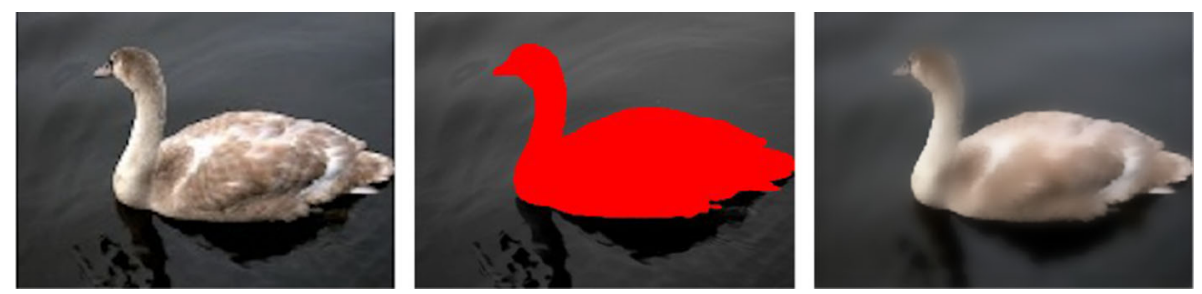

Fig. 2 Left: An image of dimensions $548 \times 402$ from the Weizmann 1-object database [2]. Middle: Two labels are used to segment the pixels. The pixels belonging to the foreground are identified by the colour-code red. Right: The image on left is filtered using a bilateral filter [50]. A bilat-

classes of methods typically optimize a cost defined on the image graph. Some commonly used segmentation methods in these categories are graph-cut [7], shortestpath segmentation [24], random walker [27], ratio cut [53], normalized cut [48,53], watershed cut [19], multicut [51] and isoperimetric partitioning [29]. For image filtering, weighted average filters such as shortest-path filters/morphological amoebas $[30,35]$, tree filter $[6,57]$ are graph based and yield good results. However, in practice, these methods cannot be applied to images with large number of pixels as their asymptotic complexity is very high.

The methods mentioned in the previous paragraph are based on cost minimization on an edge-weighted graph. Hence, the final segmentation/filtering result is a function of the edge weights of the image graph. The algorithms that are originally introduced to implement these methods take the actual weights into consideration. To ensure contrast invariance, one needs to consider only the relative ordering of the edge weights instead of the actual weights in the cost minimization. This is the core idea of the PW framework. Given a cost-minimization problem, PW framework transforms the cost-minimization problem into a series of nested cost-minimization problems. The transformation is such that the set of nested cost-minimization problems remain the same even if the edge weights of the graph are changed without altering their relative ordering. Solving the transformed problem is computationally easier than solving the original minimization problem. Additionally, the results obtained on the transformed problem are similar to those obtained on the original minimization problem. Thus, application of PW to graph-based cost minimization methods helps in scaling these methods to images with large number of pixels.

The rest of the article is organized as follows: In Sect. 2 , graphs used in image processing within the scope of the article are briefly described. Section 3 provides an explanation of the PW optimization framework in the context of image processing. The next four sections, i.e. Sects. 4, 5, 6, and 7 provide a comprehensive survey of applications of PW to classic graph-partitioning and variational cost minimization image segmentation methods. These methods are explained intuitively using toy images, simulated astronomical images, and a few eral filter suppresses the texture details within objects which are irrelevant for summarizing the information on object boundaries in the image. The boundaries between the foreground and the background are crucial for image segmentation and are preserved

figures replicated from the original articles. Section 8 contains a brief description of the utility of PW framework in explaining the links between shortest pathbased filters and spanning tree-based filters. Section 9 contains experiments on simulated astronomical sky images $[31,43]$. It is illustrated that the PW counterparts yield similar results as that of the classic methods at a lower computational cost. The conclusions section summarizes the article and provides some pointers on how to use the ideas from the article to build scalable algorithms in the context of graph-based image segmentation.

\section{Graphs in image processing}

Graphs are discrete mathematical objects. They are popularly used for data analysis as there is abundant literature available on graph algorithms. Although several variants of graph models exist, the description of graphs in this section is restricted to those essential to follow the rest of the article. The simplest of a graph model consists of two objects: a set of vertices, a subset of unordered pairs of vertices called edge set or simply edges. Two vertices are said to be adjacent to each other if the unordered pair of these vertices belongs to the edge set. In the context of images, one can identify each pixel with a vertex in the graph. As pixels in a 2D image are aligned 'nicely' like a grid, a 4-adjacency relation (see Fig. 3) is a popular choice for modelling images. In other words, each vertex is adjacent to exactly four other vertices (except at the borders of the image) viz. nearest vertices one in each of left, right, top and bottom. See Eq. 3 for a formal definition.

Each pixel has a grey value (or a triplet of values corresponding R, G, and B bands in case of colour images), one can assign a mapping on the set of vertices. However, as described in the previous section, it is convenient to model local changes. This is done by assigning weights to edges. A classic usage is to consider a discrete gradient given by the absolute difference between the pixel values (Euclidean distance between triplets) of the adjacent vertices in the case of greyscale images (colour images). This can be viewed as a dissimilarity between the neighbouring pixels (see Fig. 3). 

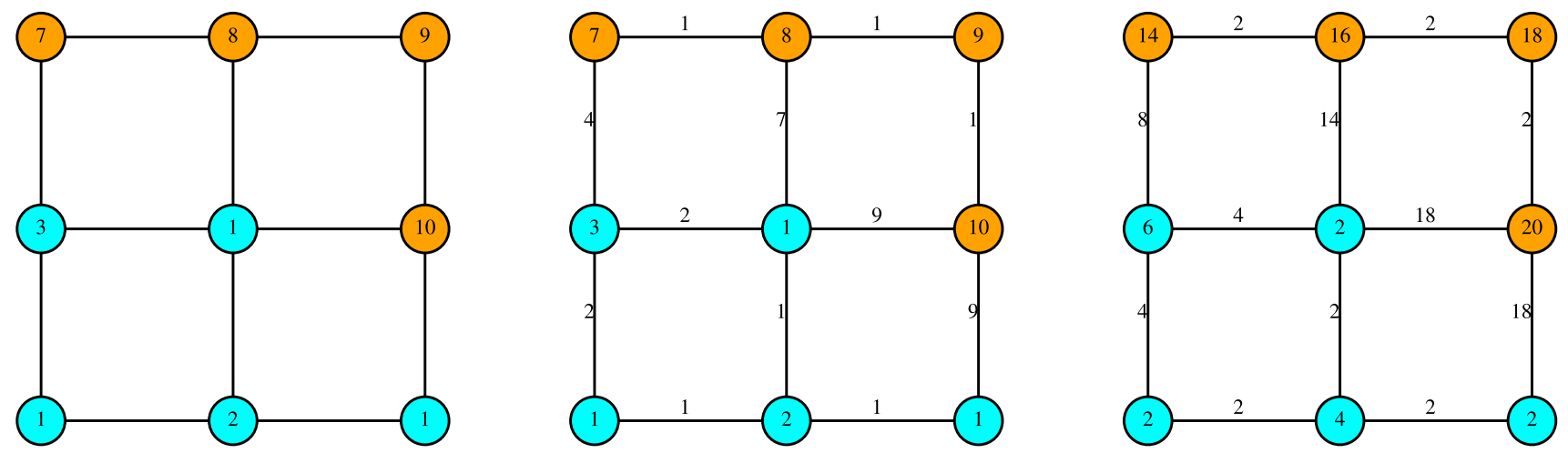

Fig. 3 Left: A toy $2 \mathrm{D}$ greyscale image of dimensions $3 \times 3$ containing two objects is represented with a vertex-weighted graph. Each pixel in the image corresponds to a vertex in the graph. The pixel intensities are displayed inside the circled vertices. Vertices are coloured with two distinct colours to signify that they belong to different objects. Middle: The image is represented as an edge-weighted graph. The

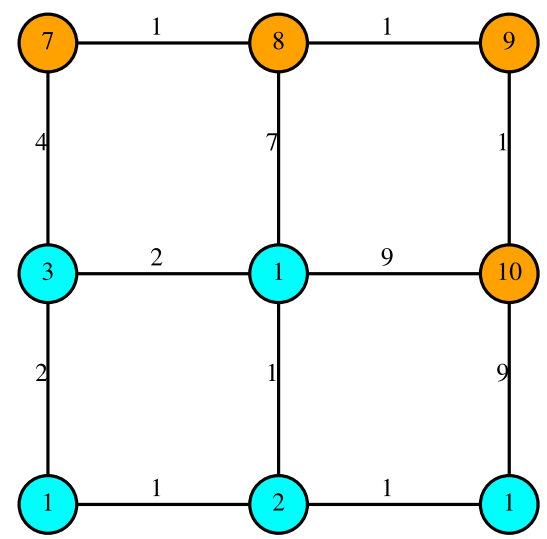

Fig. 4 Left: A toy 2D greyscale image containing two objects is visualized with a edge-weighted graph. Each pixel in the image corresponds to a vertex in the graph. The pixel intensities are displayed inside the circled vertices. Vertices are coloured with two distinct colours to signify that they belong to different objects. The edge weights are given by absolute difference of grey values of adjacent pixels. Right: The edge weights are transformed to reflect sim-

In general, if the weights of edges are constructed so as to capture dissimilarity between the adjacent vertices then the graph is said to be a dissimilarity-based edgeweighted graph. These kinds of graphs are useful for image filtering applications (see Sect. 8 for details).

On the other hand, for many image processing methods, it is convenient to capture similarity between adjacent pixels. The edge-weighted graphs with edge weights reflecting similarity between adjacent pixels are known as similarity-based edge-weighted graphs. These are particularly useful in applications where one wants to pose the objective as a cost minimization problem (Fig. 4 illustrates this fact. See Sects. 5, 6 and 7 for details). For example, if one is interested to find a segmentation of the image with a known number of segments $k$, the problem can be posed as finding a set of edges such that the sum of the weights of these edges is weights of edges are obtained by the absolute difference of image intensities and hence represent a dissimilarity measure between the neighbouring pixels. Right: A change in the contrast of the image can be represented by a scaling of the weights. Observe the intensities of the pixels and the weights of the edges are doubled

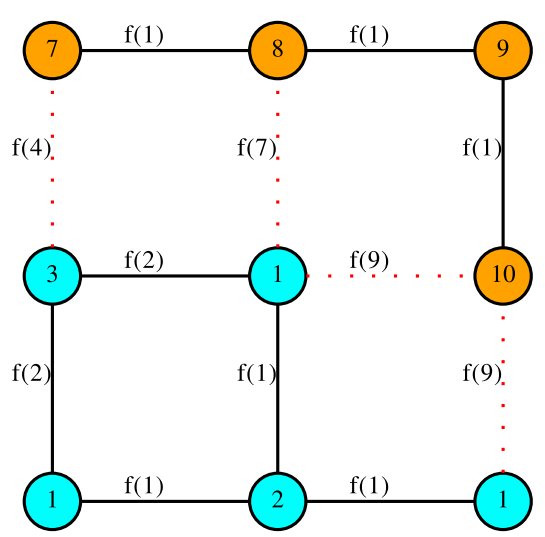

ilarities, i.e. higher the similarity, higher the weight. Here $f(x)=\exp (-x)$. Image segmentation can be posed as a minimization problem, i.e. find a set of edges such that the sum of its weights is minimum and its removal results in two pieces (as there are two objects in the image). An optimal set of edges are indicated by highlighting them as dotted edges

minimum and their removal results in $k$ pieces (called 'components' in graph theory terminology). This yields good results in practice as most of the pairs of vertices corresponding to low-weight edges w.r.t. a similarity measure belong to different objects. Figure 4 illustrates for $k=2$, also known as a graph-cut problem. In general, an image contains more than two objects and the corresponding mathematical generalization of the graph partitioning is referred to as a $k$-way cut [51].

On some occasions, prior information in the image is available. Labels of some of the pixels might be known. For example, in an astronomical sky image, some background pixels and some pixels corresponding to galaxies/stars can be identified easily. Extreme values along with low variability in the neighbourhood (see Fig. 8 in Sect. 4 for an illustration on marking seeds) are indicators for such pixels in the case of sky images. A basic 
yet important task is to segment the galaxies/stars from the background. The pixels with known labels are called seeds in image segmentation terminology. When seeds are available, one can compute the affinity between each pixel and the labelled seeds. Then, the non-seed pixels can be assigned a label corresponding to the label with maximum affinity. More often than not, these affinities are computed using 'paths' that start at the nonseed pixels and terminate at seeds. Recall that a 'path' is a sequence of distinct vertices such that every pair of consecutive vertices in the sequence are adjacent to each other. Random Walker segmentation (see Sect. 4) is based on this idea.

There are other kinds of prior information on the image. For example, it might be known a priori that certain set of pairs of pixels have different labels. This information can be imposed as a constraint on the graph partitioning problem thus making it a constrained optimization problem. Multi-cut partitioning [51] is based on this idea. More generally, on some occasions, it is known that certain pairs have the same labels (similar or attractive) and certain other pairs have different labels (dissimilar or repulsive) with varying levels of confidence. A forbidden pair of pixels having the same label can be seen as a dissimilar pair with infinite affinity. Similarly, a pair constrained to have the same labels can be visualized as a similar pair with infinite affinity. It is important to note that these pairs are not necessarily adjacent pixels. Hybrid edge-weighted graph models capturing both similarity and dissimilarity are used to model such situations. We remark that such graphs have additional edges on top of the four neighbours that each pixel has, to capture the attractions and repulsions (see Fig. 5). Each edge in the graph is either a similarity edge or a dissimilarity edge but not both. An indicator function on the edge set is used to identify whether an edge indicates a similarity or a dissimilarity. Each edge has a weight and the weight of the edge indicates the confidence on the affinity. For example, a dissimilarity edge with a high value indicates that the pair of vertices on the edge are likely to have different labels. Mutex watershed is an algorithm based on this idea. Muti-cut is closely related to mutex watershed $[54,56]$ and the reader may refer to Sect. 7 for details.

In the context of astronomical image processing, domain knowledge of the physical characteristics of the images can be incorporated into the weights of edges in various forms: similarity, dissimilarity or a combination of both similarity and dissimilarity.

\section{Power watershed optimization and contrast invariance}

In Sect. 2, we mentioned that edge-weighted graphs are well suited to image processing tasks. Specifically, three types of edge-weighted graphs have been described. These are dissimilarity-based, similarity-based, and

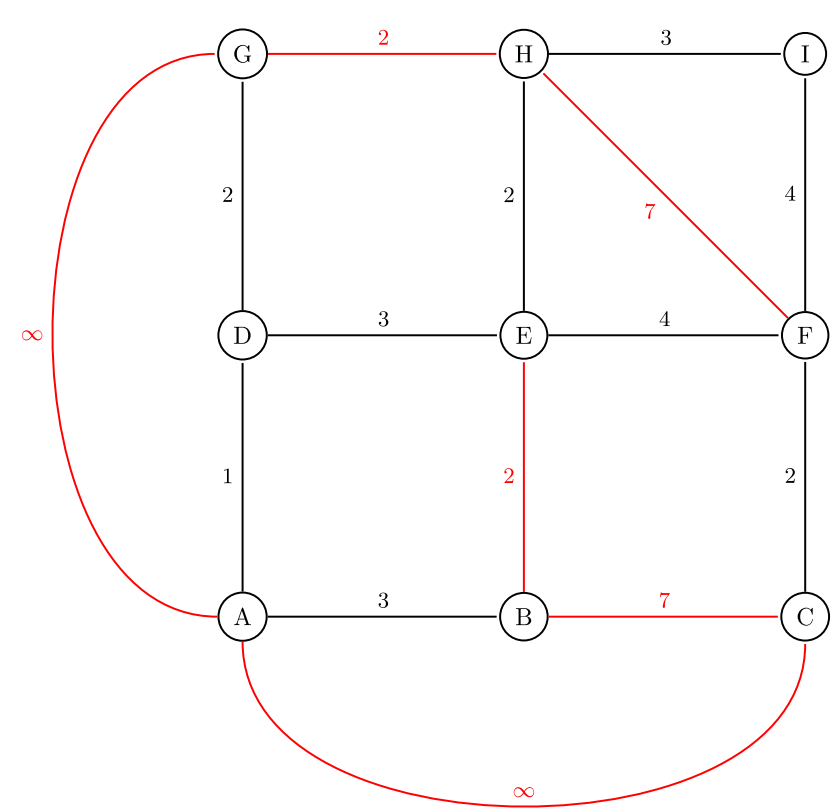

Fig. 5 Left: A hybrid edge-weighted graph capturing similarities, dissimilarities along with pairs of pixels that are forbidden to have the same labels and pairs of pixels that are required to have the same labels. A red coloured edge indicates dissimilarity and a black coloured edge indicates a similarity. The weights displayed on the edges reflects the strength of affinity. For example, a red coloured edge of value $\infty$ indicates a pair that is forbidden to have same labels

hybrid edge-weighted graphs capturing both similarity and dissimilarity. Assume that the objective i.e. image segmentation or filtering is cast as a cost minimization problem on the image graph. Further, assume that the cost can be written as a linear combination of edge weights. Allowing a slight generalization, we have the following form for the cost function $Q(\mathbf{x})$ :

$$
Q(\mathbf{x})=\sum_{e_{i j} \in E} f\left(w_{i j}\right) Q_{i j}\left(x_{i}, x_{j}\right)
$$

where $E$ is the set of edges of the image graph, $e_{i j}$ is the edge connecting the vertices $i$ and $j, w_{i j}$ is the weight of $e_{i j}, f$ is a known monotonic function (either an increasing or a decreasing function) and $Q_{i j}()$ is a smooth function (differentiable). Here $\mathbf{x}$ is a vector of size equal to the number of pixels and represents the set of target labels/values.

PW framework considers the following sequence of optimization problems:

$$
Q^{(p)}(\mathbf{x})=\sum_{e_{i j} \in E}\left(f\left(w_{i j}\right)\right)^{p} Q_{i j}\left(x_{i}, x_{j}\right)
$$

where $p$ is a positive integer and the other quantities are same as that of Eq. 5. Instead of minimizing the cost for each $p$, PW framework considers the limit of minimizers of a sequence of cost functions $\left(Q^{(p)}(\mathbf{x})\right)_{p=1}^{\infty}$ as $p \rightarrow \infty$, termed as PW limit. 
An image graph is finite, so the number of edges is finite. Further, let the set of edges, $E$ be relabelled in the increasing order of their weights. The $Q_{i j}$ terms corresponding to identical weights can be merged. The cost function can then be as sum of $l$ terms where $w_{1}<$ $\cdots<w_{l}$ are the distinct set of weights $(l \leq|E|$ holds for obvious reasons). It is shown in [39] that the limit of the minimizers can be obtained by the following algorithm:

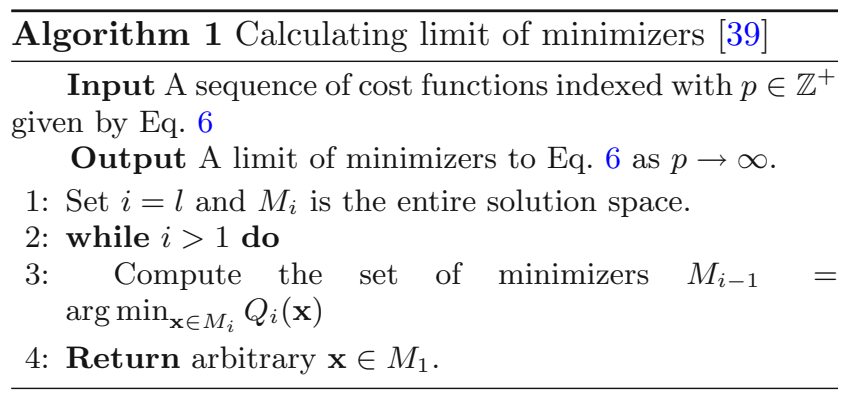

To better understand the intuition behind the algorithm, consider the following example (modified from $[39])$. Let $w>0$ and $Q^{(p)}: \mathbb{R}^{2} \rightarrow \mathbb{R}$ be defined as

$$
Q^{(p)}\left(x_{1}, x_{2}\right)=w_{1}^{p}\left(\left(x_{1}-1\right)^{2}+x_{2}^{2}\right)+w_{2}^{p}\left(x_{1}-x_{2}\right)^{2},
$$

where $w_{1}=w$ and $w_{2}=2 w$. Observe that $Q^{(p)}$ is a non-negative function for any $p>0$. The minimizer of $Q^{(p)}$ can be computed directly and is given by

$$
\begin{aligned}
& {\hat{x_{1}}}^{(p)}=\frac{2^{p}}{2^{p+1}+1} \\
& {\hat{x_{2}}}^{(p)}=\frac{2^{p}+1}{2^{p+1}+1} .
\end{aligned}
$$

With simple calculus, it is easy to verify that the sequence of minimizers of $Q^{(p)}$ i.e. $\left((\hat{\mathbf{x}})^{(p)}\right)_{p>0}=$ $\left(\left({\hat{x_{1}}}^{(p)},{\hat{x_{2}}}^{(p)}\right)\right)_{p>0}$ converges to $\left(\frac{1}{2}, \frac{1}{2}\right)$ as $p \rightarrow \infty$.

On the other hand, application of Algorithm 1 translates to rewriting $Q^{(p)}\left(x_{1}, x_{2}\right)$ as follows:

$$
\frac{Q^{(p)}\left(x_{1}, x_{2}\right)}{w_{2}^{p}}=\frac{\left(\left(x_{1}-1\right)^{2}+x_{2}^{2}\right)}{2^{p}}+\left(x_{1}-x_{2}\right)^{2} .
$$

This quantity behaves like the function

$$
\left(x_{1}-x_{2}\right)^{2} \text {, }
$$

as $p \rightarrow \infty$

At the first pass, our search space of solutions is restricted to the subspace $\left\{\left(x_{1}, x_{2}\right) \in \mathbb{R}^{2} \mid x_{1}=x_{2}\right\}$. At the next pass, within the restricted subspace of the solutions, the point that minimizes $\left(\left(x_{1}-1\right)^{2}+x_{2}^{2}\right)$ is easily seen to be $\left(\frac{1}{2}, \frac{1}{2}\right)$.

Recall that to ensure contrast invariance, the optimization problem has to remain the same irrespective of changes to the edge weights subject to preserv- ing their relative ordering. It can be seen from Algorithm 1 that the limit of minimizers of $Q^{(p)}(\mathbf{x})$ depends only on the relative ordering of the edges and not the actual weights. Also, it is worth noting that Algorithm 1 decomposes the cost function on the original graph into costs on smaller subgraphs. It is shown in [10 $12,17,18,21-23]$ that the computation of said limit is easier than minimizing the original cost $Q(\mathbf{x})$. In Sects. $4,5,6$ and 7 , it will be demonstrated that the quality of the segmentation is retained while reducing the computational cost.

It is important to mention that the $\mathrm{PW}$ limit of a cost of the form Eq. 5 depends only on a substructure of the image graph (known as a subgraph in graph theory). This subgraph is either union of maximum spanning trees (UMaxST) or the union of minimum spanning trees (UMinST) depending on whether the edge weights reflect similarity or dissimilarity. UMaxST (respectively, UMinST) is the induced subgraph generated by set of edges that belong to at least one 'maximum spanning tree' (respectively 'minimum spanning tree') of the image graph. Recall that a 'spanning tree' is a subset of edges of the image graph such that there exists a unique path between every two vertices in the image graph when the paths are restricted to contain only edges from the subset of edges (see Fig. 6 for an illustration). A maximum spanning tree (MaxST) is a spanning tree such that no other spanning tree has the sum of its edge weights larger than that of MaxST. A similar definition holds for a minimum spanning tree (MinST).

\section{Fast random walker segmentation}

Recall that in some practical applications, prior information of labels of some pixels in the image is available. Random walker is a seeded segmentation method and is handy for such situations.

\subsection{Classic random walker segmentation}

Random walker (RW) can be described as follows: A similarity-based edge-weighted graph is constructed on the image. For each non-seed, several random walks are simultaneously propagated to each of its neighbours and the process is recursively repeated. At each step, it is assumed that a random walk splits into multiple unvisited vertices independently. Further, the branching probability of an edge on a vertex is equal to the proportion of similarity of the edge traversed to the sum of similarities of all possible edges that can be traversed at that step. For example, in Fig. 7, a random walker starting at vertex 1 selects the edge 1-2 with probability $\frac{2}{3}$ as there are two edges emanating from 1 namely 1-2 with weight 2 and 1-4 with weight 1 . These paths are recursively propagated until they terminate on labelled points, i.e. seeds. For example, a random walker starting at the vertex 1 that already chose the first edge as $1-2$ will choose the edge $2-3$ with condi- 

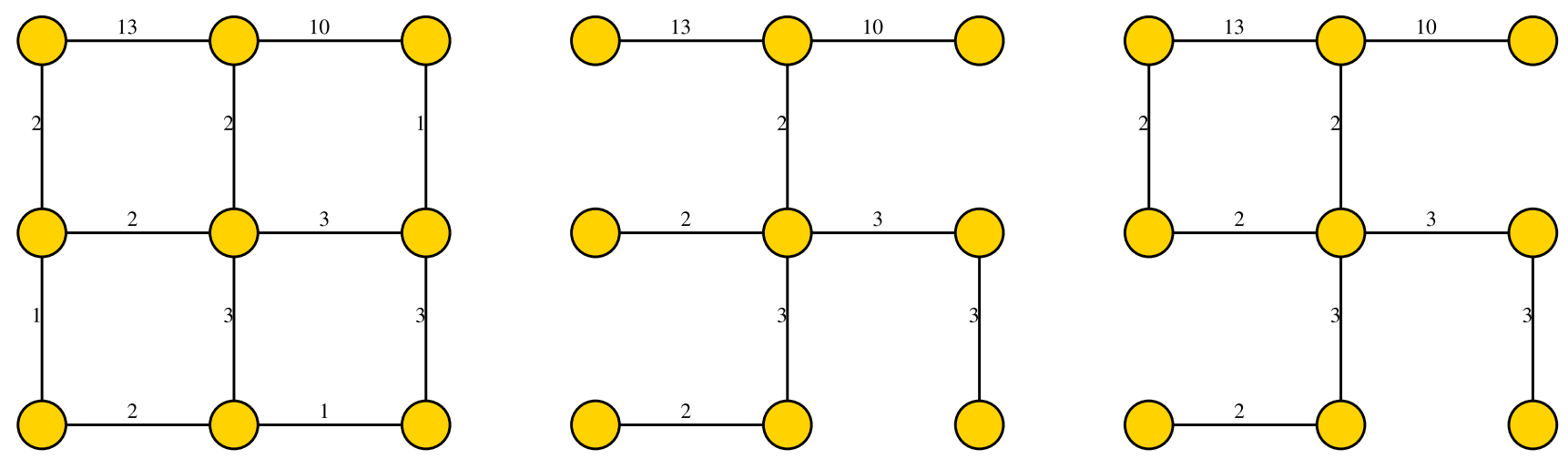

Fig. 6 Left: An edge-weighted graph. Middle: A MaxST of the graph on left. Right: UMaxST of the graph on left
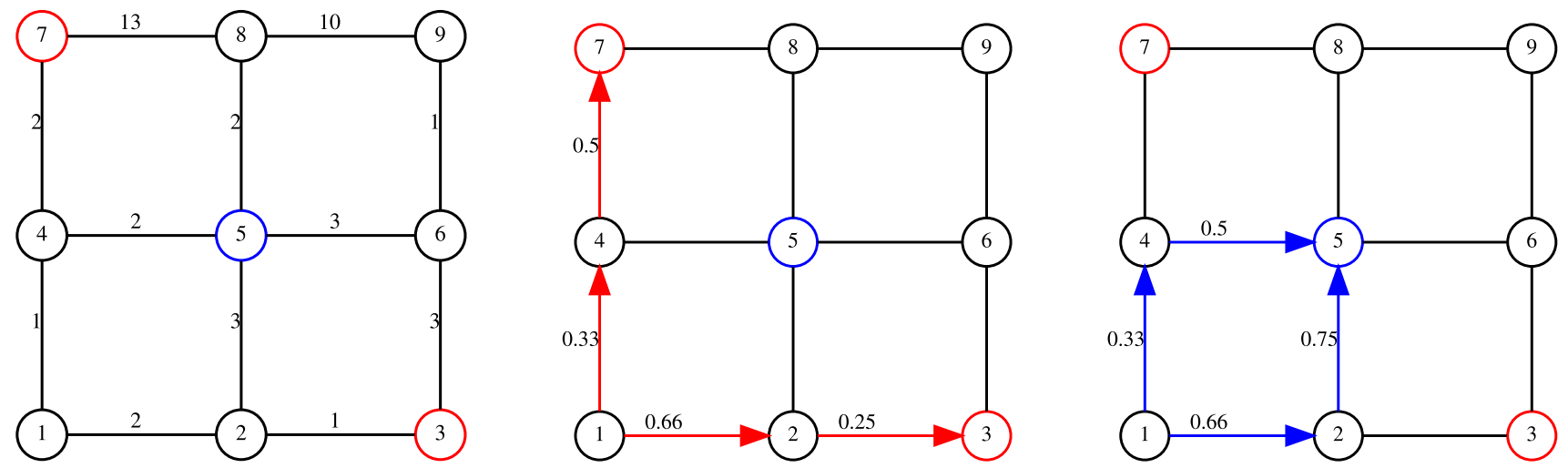

Fig. 7 Left: A similarity-based edge-weighted graph with two labels coloured blue and red with three seeds, two seeds with colour red, and one seed with colour blue. Middle: The paths from vertex 1 to seeds with red label are highlighted in red. Random walk probabilities are displayed on the corresponding edges. The probability that the vertical path from vertex 1 to red seed vertex 7 is $0.33 \times 0.5$. Similarly, the prob-

tional probability $\frac{1}{4}$. Note that the random walker has only two choices of edges namely $2-5$ and $2-3$ at this stage as vertex 1 is already visited. The probability of a non-seed pixel having a given label is then calculated by summing up probabilities of all the paths that start from the non-seed pixel to all seeds with that label (see Fig. 7 for an illustration). A non-seed is then assigned the label to which the RW probability is highest. For example, in Fig. 7, vertex $N$ would be assigned label $B$.

Recall that a seeded classification problem with multiple labels can also be perceived as a collection of binary classification problems. The number of such binary classification problems would be as many as the number of distinct labels. Let $\mathcal{L}$ denote the set of distinct labels. Each such binary classification problem deals with identifying label $l$ versus NOT label $l$, where $l \in \mathcal{L}$ is a label. Treating the multiple class classification problem this way, it was shown in [27] that RW probabilities of each such binary classification problem can be obtained by minimizing the convex cost function given by Eq. 12 . abilities are computed for the horizontal path from 1 to 3 which is also a red seed. Adding these probabilities yields the RW probability for vertex 1 to be labelled red. Left: The paths from 1 to seed with the label blue are highlighted. The path probability computations are performed similarly. The RW probability for the vertex to be labelled either red or blue would add up to 1

$$
\begin{aligned}
\operatorname{RWCost}(\mathbf{x}) & =\frac{1}{2} \sum_{e_{i j} \in E} w_{i j}\left(x_{i}-x_{j}\right)^{2}, \text { subject to } \mathbf{x}_{\text {seed }} \\
& =\mathbf{f}_{\text {seed }},
\end{aligned}
$$

where vector $\mathbf{x}$ refers to the target labels of all pixels, $E$ is the set of edges, $w_{i j}$ is the weight of $e_{i j}, \mathbf{x}_{\text {seed }}$ refers to the target labels of seed pixels and $\mathbf{f}_{\text {seed }}$ is the vector of preset labels of corresponding seeds. Note that $\mathbf{f}_{\text {seed }}$ is a vector of zeros and ones as we are dealing with a binary classification problem. More particularly, in the $l$ versus NOT $l$ classification problem, a coordinate in $\mathbf{f}_{\text {seed }}$ equals 1 if the corresponding pixel is labelled $l$ and 0 if it is labelled but the corresponding label is NOT $l$.

Let $L$ denote the unnormalized Laplacian of the graph [53]. One can write the cost RWCost( $\mathbf{x})$ in Eq. 12 as $\mathbf{x}^{T} L \mathbf{x}$ up to a constant factor. Rearranging the indices of seeds and non-seeds so that the indices of all seeds appear before those of non-seeds, one can decompose $L$ as follows: 

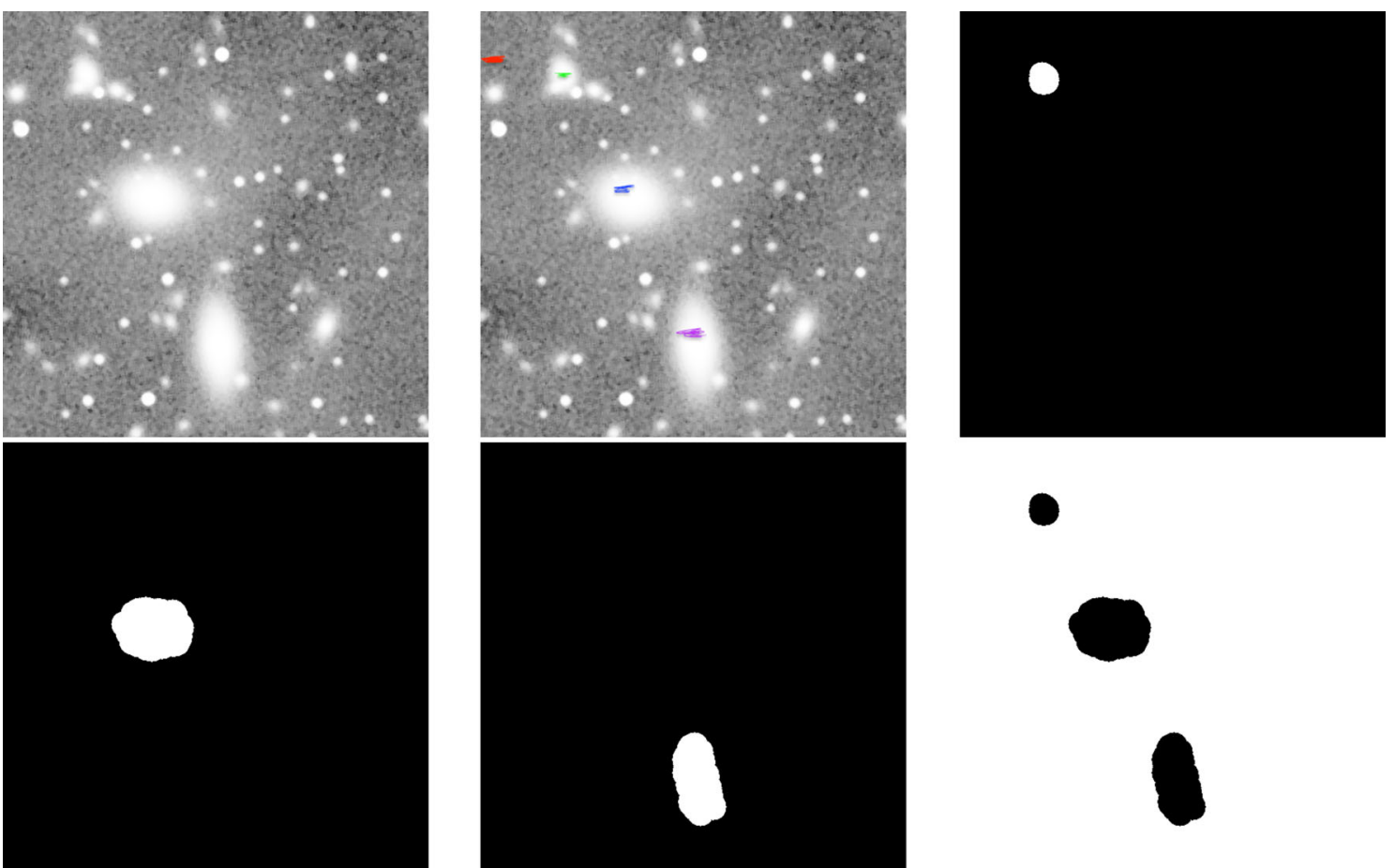

Fig. 8 Top left: An image to be segmented. Top middle: Seeds are marked with strokes. Four different labels are used for RW segmentation. Top left: Visualization of RW Probability map of green label. Bottom: From left to right, each

$$
L=\left(\begin{array}{cc}
L_{\text {seed }} & B \\
B^{T} & L_{U}
\end{array}\right)
$$

RWCost $(\mathbf{x})$ can then be written as

$$
\begin{aligned}
\operatorname{RWCost}(\mathbf{x})= & \frac{1}{2}\left(\mathbf{x}_{\text {seed }}^{T} L_{\text {seed }} \mathbf{x}_{\text {seed }}\right. \\
& \left.+2 \mathbf{x}_{U}^{T} B^{T} \mathbf{x}_{\text {seed }}+\mathbf{x}_{U}^{T} L_{U} \mathbf{x}_{U}\right)
\end{aligned}
$$

where $\mathbf{x}_{U}$ denotes the sub-vector of $\mathbf{x}$ corresponding to the unlabelled points. Applying elementary calculus, it can be seen that the solutions to the minimization problem can be obtained by solving the following linear system of equations:

$$
L_{U} \mathbf{x}_{U}=-B^{T} \mathbf{x}_{\text {seed }}
$$

Recall that the number of distinct labels in the multiple class classification problem is $|\mathcal{L}|$. Hence, one needs to compute an affinity vector of length $|\mathcal{L}|$ to encode probabilities of the unlabelled points to each of the distinct labels. In other words, Eq. 15 transforms to solving

$$
L_{U} X=-B^{T} S
$$

where $X$ is a matrix of shape $\left|\mathbf{x}_{U}\right| \times|\mathcal{L}|$ and $S$ is a matrix of size $\left|\mathbf{x}_{\text {seed }}\right| \times|\mathcal{L}|$.

An application of RW on a real image is illustrated in Fig. 8 (this demonstration is similar to the illustration on the medical image from the original paper on RW $[27])$. image is a visualization map of label RW probabilities for labels blue, violet and red in that order. White shades indicate high probability and dark indicate low probability

\subsection{Power watershed approximation to random qalker}

It can be seen that Eq. 12 is in the form of Eq. 5 . In $[17,18]$, the RW cost function is recast in the form of Eq. 6 using the PW framework. The cost minimization is transformed into a family of cost minimization problems as given by Eq. 17 indexed by $p$ where $p \in \mathbb{N}$.

$$
\begin{aligned}
\operatorname{RWCost}^{(p)}(\mathbf{x})=\frac{1}{2} & \sum_{e_{i j} \in E} w_{i j}^{p}\left(x_{i}-x_{j}\right)^{2} \\
& \text { subject to } \mathbf{x}_{\text {seed }}=\mathbf{f}_{\text {seed }} .
\end{aligned}
$$

It was shown in [18] that the results obtained by the limit of minimizers to Eq. 17 as $p \rightarrow \infty$ are comparable to RW at the benefit of lesser computational cost. Recall Algorithm 1 from Sect. 3. The limit of minimizers to Eq. 17 as $p \rightarrow \infty$ is computed as follows - first, the sum of terms corresponding to the highest weights in the summation of Eq. 17 are considered. This summation is minimized subject to the seed constraints. The solution set is then restricted to those obtained at this level and the sum of terms corresponding to the next highest weight is considered. This summation is minimized subject to seed constraints and the solution set constraint obtained from the minimization problem(s) at the higher weights. The sum of terms corresponding to the next highest weights are then considered. This 

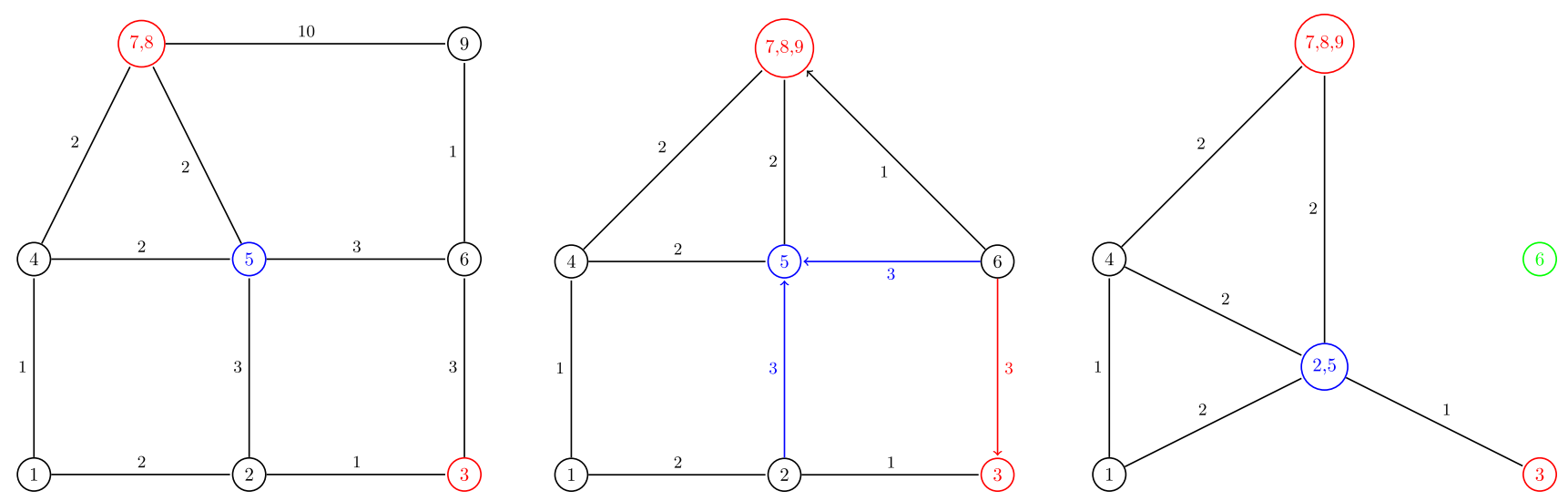

Fig. 9 Consider the toy image from Fig. 7. This figure shows the application of PW on RW. Left: After first step of the PW sequential optimization of Eq. 17, the graph is collapsed to a smaller graph. Vertex 8 is then labelled red. Middle: After the second step in PW optimization, the graph collapses further as shown. At this stage, vertex 9 is also

process is repeated until all the distinct weights are processed.

In case of random walker, this process can be envisioned as follows: consider the subgraph of the image graph with only the highest weights. Examine each of the connected components of this graph. If there is no seed in a connected component, the connected component is collapsed and the target labels of each of the vertices in the connected component are constrained to be same. If there is exactly one seed in a connected component, the connected component is collapsed and the target labels of each of the vertices in the connected component are set as the label of the seed. In case of two or more seeds in a connected component, a random walker is solved on the connected component using Eq. 16 as described in Sect. 4.1. The collapsed graph is considered for the sequential processing. The pixels that are already labelled are also treated as seeds for the subsequent steps. Next, the edges of second highest weight are considered and the process is repeated. This process is continued until all the edges of the image graph are processed. Figure 9 illustrates an example of PW approximation to RW. A formal algorithm to solve a random walker segmentation with two labels is given by Algorithm 2. For more than two labels, the idea is similar and one needs to work with one-hot encoding to obtain probabilities.

Essentially, the application of PW implies that the linear system of equations used for computing random walk probabilities have to be solved on subgraphs of the image graph sequentially. In general, the sizes of the subgraphs obtained at each step are not deterministic in nature. However, typically these subgraphs end up being very small when compared to the image graph. This would significantly reduce the computational cost. Figure 10 from [18] shows a comparison of the time taken to obtain segmentation results on 2D images [44] as a function of number of pixels by random walker labelled red. Using the next highest weights of magnitude 3 , random walks are illustrated in respective colours to the labels. Left: After solving the RW in the middle figure, the probabilities that vertex 6 is labelled red and blue are computed (probabilities are not shown in the figure). In the next step, the graph is further collapsed and is illustrated here $\overline{\text { Algorithm } 2 \text { PW Approximation to Random Walker }}$ Segmentation [18]

Input An edge-weighted graph $\mathcal{G}=(V, E, W)$ and seeds with two labels $x_{i}=1$ for $i \in F$ and $x_{j}=0$ for $j \in B$, $F, B \subset V$ with $F \cap B=\emptyset$

Output Random walker probabilities for each $x_{k}$ for $k \in V \backslash(F \cup B)$

1: Sort the edge set $E$ in decreasing order of weights.

2: Decompose $E$ into sets of edges with different weights $E=\cup_{i=1}^{l} E_{l}$ where $E_{r}$ contains edges with weight $w_{r}$ for each $1 \leq r \leq l$ with $w_{r}>w_{r^{\prime}}$ if $r>r^{\prime}$.

3: Denote $V_{r}$ as the set of vertices that are incident on $E_{r}$ for each $1 \leq r \leq l$. Set $i=l$.

4: while $i>\overline{0}$ do

5: $\quad$ Solve Eq. 12 on the graph $\mathcal{G}_{i}=\left(V_{i}, E_{i},\left.W\right|_{E_{i}}\right)$ on each connected component separately with known label probability then

7: $\quad$ Collapse the vertices of the connected component into a single vertex. The label probability values will be identical on each of these vertices eventually when computed

8: $\quad$ else

9: The solver yields fixed label probabilities. Consider all the label probabilities known for these vertices

10: $\quad$ Set $i=i-1$

11: Return label probabilities $x_{i}$ for each $i \in V$.

algorithm (in green), PW approximation to random walker (in red) along with a few other popular segmentation methods. It can be seen that for images with relatively larger number of pixels, the PW approximation to RW scales well. The downside is that there are no theoretical guarantees on the approximation to the optimal solution.
6: if Connected component does not contain any vertex 


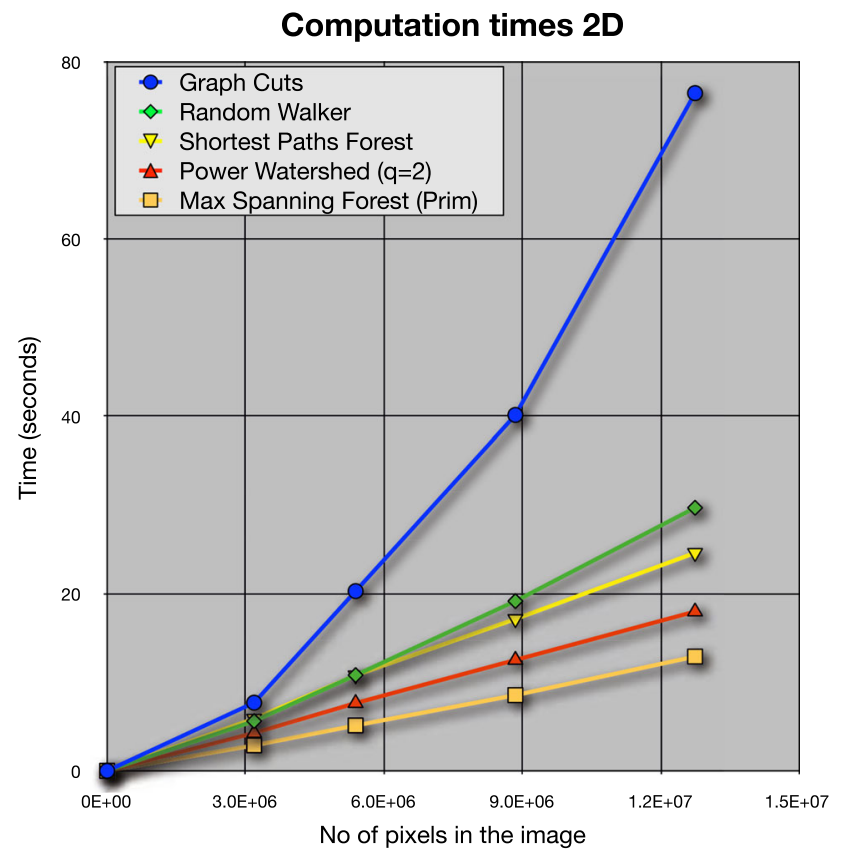

Fig. 10 Comparison of computation times of 2D seeded image segmentation methods on [44] (this figure is replicated from [18]). For each dimension, the times were generated by segmenting the same image scaled down. Power watershed $(q=2)$ corresponds to the power watershed approximation to random walker (in red) and random walker is highlighted in green. Notice that for images with relatively larger number of pixels, the difference in time taken is significant

\section{Fast and robust isoperimetric segmentation}

Recall from Sect. 2 that a graph-cut separates the graph into two components by removing a set of edges. The sum of weights of these edges is minimum among all such sets whose removal results in breaking the graph into two components. Mathematically, it boils down to minimizing the following quantity over all subsets $A$ of the vertex set.

$$
W(A, \bar{A})=\sum_{e_{i j} \in E, i \in A, j \in \bar{A}} w_{i j},
$$

where $E$ denotes the set of edges of the graph and $w_{i j}$ denotes the weight of edge $e_{i j}$. The summation runs over all edges such that one endpoint of the edge is in $A$ and the other in $\bar{A}$.

Each of these components can be viewed as an object. The sum of edge weights is likely to be less when the edges removed are less in number. In practice, more often than not, a graph-cut results in small components as removal of a few edges is sufficient to separate the graph into a small and a big component (see Fig. 11). Small components are not desired as they practically do not represent any meaningful objects. One of the common approaches to avoid small components is to impose a penalty on the size of the components in the cost func- tion. Ratio cut $[8,53]$, normalized cut [53], and isoperimetric cut $[28,29,36]$ are some of the popular methods that impose a penalty on the size. Isoperimetric cut can be described as the following cost minimization problem:

$$
\operatorname{Iso} \operatorname{Cost}(A)=\frac{W(A, \bar{A})}{\min \{|A|,|\bar{A}|\}},
$$

where $A$ is a subset of the vertices and $\bar{A}$ denotes its complement. The numerator in the cost is the cost function minimized by graph cut (see Eq. 18). The denominator penalizes the cost on small components so that minimizing the cost does not yield them. However, this is an NP-hard problem.

\subsection{Classic approach to isoperimetric graph partitioning for image segmentation}

Notice that the numerator in Eq. 19 can be rewritten as

$$
\begin{aligned}
W(A, \bar{A}) & =\sum_{e_{i j} \in E, i \in A, j \in \bar{A}} w_{i j} \\
& =\sum_{e_{i j} \in E} w_{i j}\left(x_{i}-x_{j}\right)^{2}
\end{aligned}
$$

where $x_{i}=1$ if $i \in A$ and $x_{i}=0$ if $x_{i} \in \bar{A}$. Using the notion of unnormalized graph Laplacian [53], one can compactly rewrite the cost function in $\mathrm{Eq} 19$ as

$$
\operatorname{IsoCost}(A)=\frac{\mathbf{x}^{T} L \mathbf{x}}{\min \left\{\mathbf{x}^{T} \mathbf{1},(\mathbf{1}-\mathbf{x})^{T} \mathbf{1}\right\}},
$$

where $\mathbf{x}$ is the vector of vertex labels, i.e. $x_{i}=1$ if $i \in A$ and $x_{i}=0$ of $i \in \bar{A}$. $L$ is the unnormalized graph Laplacian.

Typically, to obtain an approximation solution to NP-hard problems with a discrete constraint set, the constraints are relaxed. The transformed problem is known as a continuous relaxation of the corresponding NP-hard problem. The solutions to continuous relaxation can be obtained easily. All possible thresholds of such solutions are examined and an optimal threshold, i.e. the discretized solution with lowest cost is identified as a heuristic approximation. In practice, such heuristics work well. However, in this particular case, allowing continuous relaxation without further constraints would lead to meaningless solutions. This is because of the cost in Eq. 20 is always non-negative (a graph Laplacian is a positive semi-definite [53]). For the relaxed constraints, the cost can be made arbitrarily close to zero (which would be the minimum cost) for every possible partition of the graph (see [22] for details). Hence, an additional constraint is added, i.e. the label of an arbitrary vertex $r$ is set to be zero without loss of generality. The transformed problem can be written as 
Fig. 11 Left: A

similarity-based

edge-weighted graph.

Right: Small Cut problem

illustrated. Eight vertices

in one component and one vertex in the other resulted by graph-cut. The cut edges are highlighted as dotted red edges and the vertices belonging to different components are colour-coded
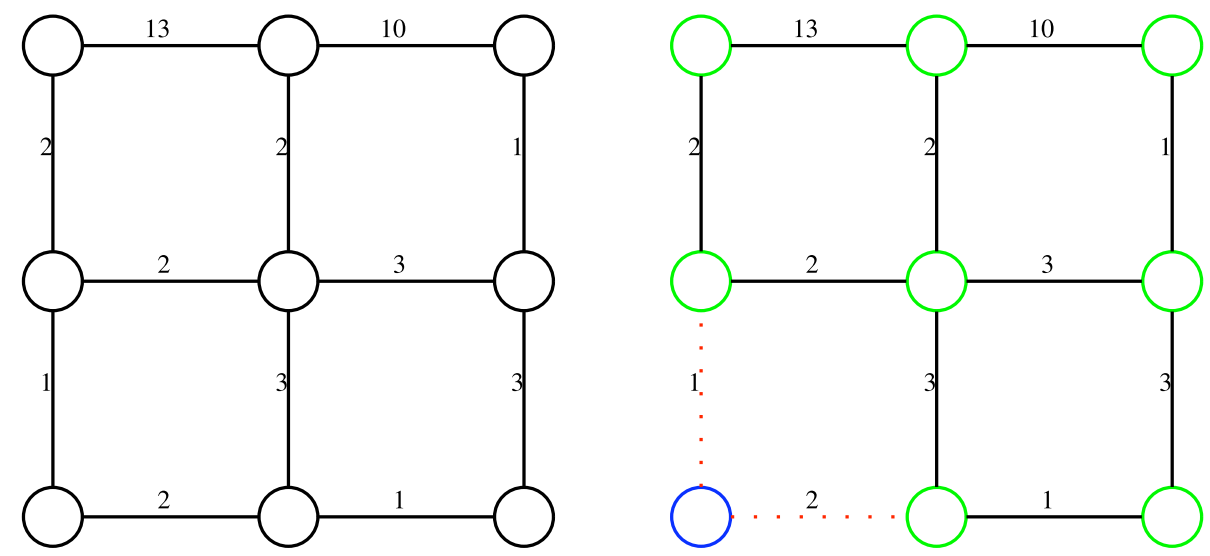

$$
\text { Minimize } \frac{\mathbf{x}_{-r}^{T} L_{-r} \mathbf{x}_{-r}}{\min \left\{\mathbf{x}_{-r}^{T} \mathbf{1},\left(\mathbf{1}-\mathbf{x}_{-r}\right)^{T} \mathbf{1}\right\}},
$$$$
\text { subject to each } x_{i} \in[0,1] \text {, }
$$

where $\mathbf{x}_{-r}$ denotes the vector $\mathbf{x}$ with the $x_{r}$ removed and $L_{-r}$ denotes $L$ after deletion of $r$ th row and columns.

Using Lagrange multipliers, solving Eq. 21 is equivalent to solving:

$$
L_{-r} \mathbf{x}_{-r}=\mathbf{1} .
$$

The reader may refer to Fig. 24 in Sect. 9 for an illustration of segmentation on a simulated astronomical image obtained by solving the relaxed isoperimetric partitioning.

\subsection{Spanning tree-based approaches to isoperimetric graph partitioning}

In [26], it was suggested that solving the continuous relaxation problem on a MaxST of the image graph scales well. To elaborate, recall that the average number of non-zero, non-diagonal elements in each row of a graph Laplacian is the average degree of a vertex in the graph (number of neighbours of a vertex is known as the degree of the vertex in graph theory). For example, from 2D images to 3D images, the sparsity of the graph Laplacian decreases due to an increase in average degree from four to six. Hence, solving the linear system in Eq. 22 is slower on 3D images and can be prohibitively slow for denser graphs. On the other hand, the graph Laplacian of a MaxST always has an average of two non-zero, non-diagonal elements in a row. Also, the results obtained by solving the continuous relaxation on an arbitrary MaxST were shown to be reasonably good in terms of quality for 3D medical image segmentation.

Later, in [22] it was shown that the solutions obtained by solving Eq. 22 on an arbitrary MaxST are not consistent with those solved on the image graph (see Fig. 12 replicated from [22]). It was established in [22] that application of PW framework theoretically implies that it is enough to solve Eq. 22 on UMaxST of the image graph. Algorithm 3 provides a formal algorithm to obtain the $\mathrm{PW}$ approximation to the isoperimetric graph partitioning.

$\overline{\text { Algorithm } 3 \mathrm{PW} \text { Approximation to Isoperimetric }}$ Graph Partitioning [22]

Input An edge-weighted graph $\mathcal{G}=(V, E, W)$

Output A heuristic approximation to the isoperimetric graph partition i.e. labels $x_{i}=0$ or $x_{i}=1$ for each $i \in V$.

1: Choose an arbitrary vertex $r \in V$. Set $x_{r}=0$

2: Compute the UMaxST of $\mathcal{G}$. Denote it by $\mathcal{G}_{\text {UMax }}$ ST

3: Compute the unnormalized graph Laplacian $L_{U M a x S T}$ of $\mathcal{G}_{U M A X S T}$.

4: Delete the $r$ th row and column of $L_{U M a x S T}$. Denote this by $L_{(U M a x S T,-r)}$.

5: Denote the vector of labels for each vertex as $\mathbf{x}$. Remove the $r$ th coordinate from $\mathbf{x}$ and denote it by $\mathbf{x}_{-r}$.

6: Solve $L_{(U M a x S T,-r)} \mathbf{x}_{-r}=\mathbf{1}$ for $\mathbf{x}_{-r}$ and denote the optimal solution by $\mathbf{x}_{-r}^{\text {opt }}$

7: Let $\mathbf{x}^{\text {opt }}$ denote the vector with $x_{r}=0$ inserted at $r^{\text {th }}$ coordinate.

8: Compute all possible thresholds of $\mathbf{x}^{\mathrm{opt}}$, check the cost obtained by each such threshold solution using Eq. 20.

9: Return the solution corresponding to the lowest cost in the previous step.

Further, it was supported by empirical evidence that these solutions are consistent with those solving Eq. 22 on the image graph (see Fig. 12). Although the number of edges in the UMaxST varies in general and can be as large as that of the image graph itself, it was shown empirically on several $2 \mathrm{D}$ image databases that the reduction in a number of edges on an average is about 25 percent. Figure 13 from [22] shows histograms on the percentage of redundant edges removed, i.e. the edges present in the image graph but not in the UMaxST of the image graph. These edges do not contribute to the final segmentation results. However, if they are not discarded, the linear solver in Eq. 22 can be prohibitively slow especially when the number of edges are large in the image graph. This is because, the sparser the adjacency matrix of the graph, the more zeros in the corresponding Laplacian.

To summarise, the solution obtained by application of PW to the relaxed isoperimetric partitioning cost 

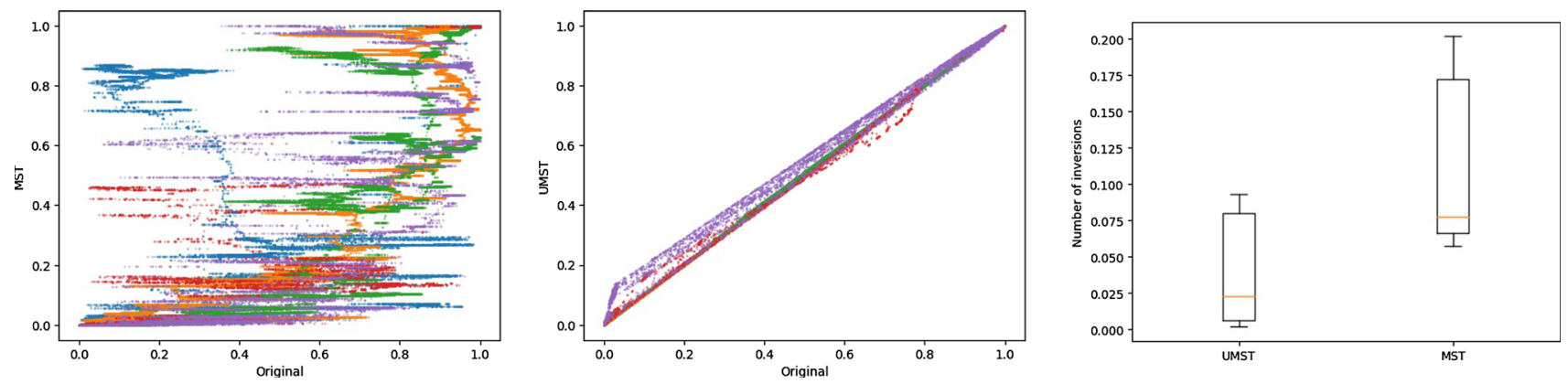

Fig. 12 The relative ordering of the solutions to the continuous relaxation problem of the isoperimetric partitioning (this figure is replicated from [22]). Each colour in the left and middle figures correspond to one image in the Weizmann 1-object dataset [2]. A strictly increasing plot implies perfectly consistent solutions w.r.t. solving Eq. 22 on the

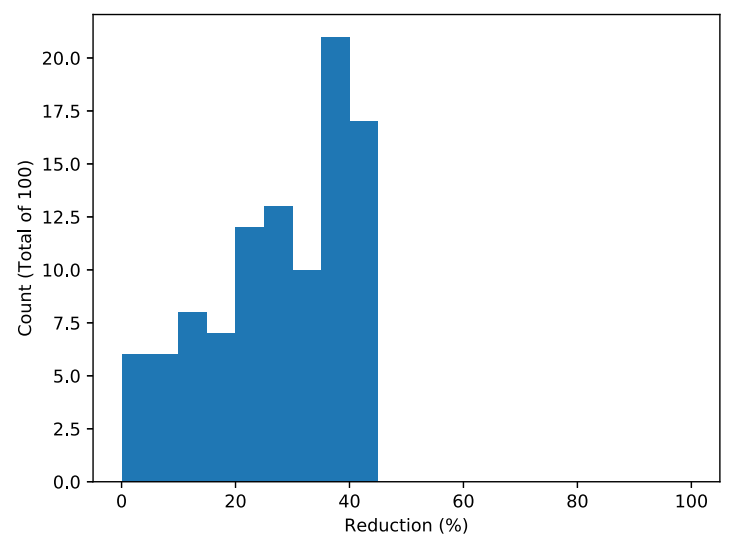

Fig. 13 Histograms indicating the percentage of redundant edges removed by UMaxST. $x$-axis represents the percentage reduction obtained (this figure is replicated from [22]). $y$-axis represents the number of images achieving the

minimization is a robust yet fast approximation to the relaxed isoperimetric partitioning cost minimization problem. In particular, the PW approximation is very useful for segmenting astronomical images which usually have very large number of pixels. The downside is that there are no theoretical guarantees on how close the PW approximation solution is from the optimal solution to the relaxed problem. For experiments on simulated astronomical images, the reader may refer to Sect. 9.

\section{Fast spectral clustering}

Ratio cut is another variant of graph-cut designed to avoid small components. Suppose we know that an image contains $k$ objects, ratio cut can be described as minimizing the following cost splitting the vertex set $V$ of the image graph into disjoint subsets $A_{1}, \ldots, A_{k}$ : image graph. In both the figures in left and middle, the $X$ axis corresponds to the image graph. Left: Arbitrary MaxST versus image graph. Middle: UMaxST versus image graph. Right: A box plot indicating the proportion of inversions on the solutions obtained by an arbitrary MaxST and UMaxST w.r.t. the solutions on image graph

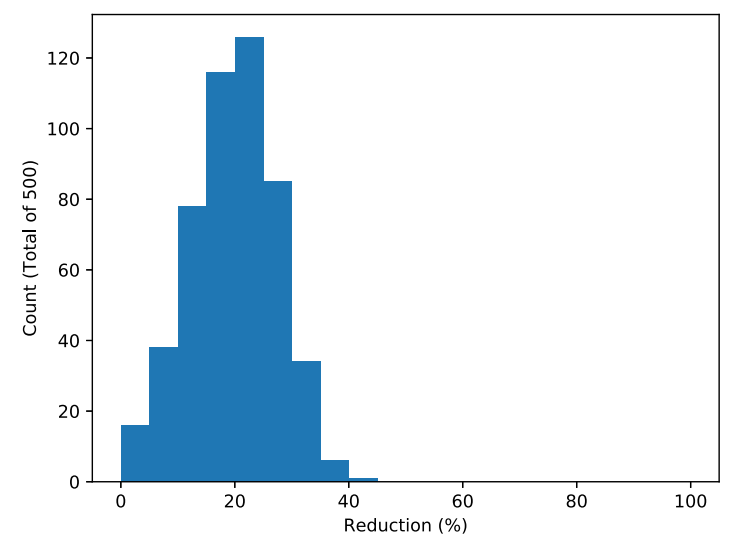

given amount of reduction. The results are computed on Left: Weizmann 2-Object dataset [2], Right: on BSDS500 dataset [3]

$$
\operatorname{RatioCost}\left(A_{1}, \ldots, A_{k}\right)=\sum_{t=1}^{k} \frac{W\left(A_{t}, \bar{A}_{t}\right)}{\left|A_{t}\right|}
$$

where $W(A, \bar{A})$ is given by Eq. 18 .

\subsection{Classic approach to ratio cut}

However, the ratio-cut problem is NP-hard. To obtain an approximation solution, typically a continuous relaxation is constructed. A basic construction is done as follows:

Set $x_{i}=\sqrt{\frac{|\bar{A}|}{|A|}}$ if $i \in A$ and $x_{i}=-\sqrt{\frac{|A|}{|\bar{A}|}}$ if $i \in \bar{A}$. These $x_{i}$ s are supposed to be visualized as onedimensional representations of the vertices. The ratiocut cost can then be rewritten as

$$
\operatorname{Ratio} \operatorname{Cost}(A, \bar{A})=\left(\frac{2}{|A|+|\bar{A}|}\right) \mathbf{x}^{T} L \mathbf{x},
$$

where $L$ is the unnormalized Laplacian of the image graph. Observe that $\mathbf{x}^{T} \mathbf{x}=|A|+|\bar{A}|=|V|$ is a constant 
by the choice of each $x_{i}$. The relaxed ratio cut problem can be expressed as

$$
\text { Minimize } \mathbf{x}^{T} L \mathbf{x} \text { subject to } \mathbf{x}^{T} \mathbf{x}=|V| \text {. }
$$

This is the classic eigenvector problem i.e. finding an eigenvector with smallest eigenvalue. It is easy to see that the ratio cut cost is always non-negative. Further, a zero cost is attained when $\mathbf{x}$ is a constant vector. However, a constant vector does provide any information on partitioning the vertices as the corresponding $x_{i}$ values are identical. Hence an additional constraint should be added to avoid constant vectors. As we are working with Eigenspaces, it is enough to search in the orthogonal space to the linear space spanned by constant vectors, i.e.

$$
\begin{aligned}
& \text { Minimize } \mathbf{x}^{T} L \mathbf{x} \text { subject to } \\
& \mathbf{x}^{T} \mathbf{x}=|V|, \text { and } \mathbf{x}^{T} \mathbf{1}=0 .
\end{aligned}
$$

In general, representations with more than one dimension carry more information. As a thumb rule, for partitioning the graph into $k$ components, a $k$ dimensional representation is used. A continuous relaxation of the ratio cut problem can thus be rewritten as the following minimization problem:

$$
\text { Minimize } \operatorname{Tr}\left(H^{T} L H\right) \text { subject to } H^{T} H=I_{k},
$$

where $\operatorname{Tr}()$ denotes the trace operator of a matrix i.e. sum of diagonal elements of a matrix, $I_{k}$ is an identity matrix of size $k, H$ denotes a $|V| \times k$ matrix with $x_{t i}=\sqrt{\frac{\left|\bar{A}_{t}\right|}{\left|A_{t}\right|}}$ if $i \in A_{t}$ and $x_{t i}=-\sqrt{\frac{\left|A_{t}\right|}{\left|A_{t}\right|}}$ if $i \in \bar{A}_{t}$. Theoretically, the solution to Eq. 27 is obtained by finding the first $k$ eigenvectors of Laplacian $L$ of the image graph.

The row vectors of $H$ obtained by solving Eq. 27 are called spectral embeddings. Simple Euclidean distances between spectral embeddings capture the objects reasonably well in an image. Typically, standard algorithms such as $k$-means [32] are applied to use these distances to obtain the final image segmentation (see Fig. 14 for an illustration on a general edge-weighted graph). It is worth mentioning that multi-scale combinatorial grouping $[4,41]$ which uses spectral clustering as a building block achieved state-of-the-art on a popular image segmentation dataset when published.

An application of ratio cut on a simulated astronomical image shown in the left image of Fig. 8 is illustrated in Fig. 15. The ratio cut is performed as follows: first, a histogram equalization is performed on the noisy image. This is followed by a median filter. A 4-adjacency similarity graph is constructed on this image. An opening is then performed on the median filtered image. On the opened image, pixels with intensities less than a low preset threshold and pixels with intensities greater than a high preset threshold are identified as two groups of pixels. Each of pixels in the first group are connected to an auxiliary vertex. Similarly, each of the pixels in the second group are connected to a second auxiliary vertex. The weights of the edges incident on either of the auxiliary vertices are set to the same value slightly larger than the highest weight among the edges of the 4 -adjacency graph. A ratio cut is then performed on this graph with $k=2$ clusters. The auxiliary vertices are then discarded and the labels of the other vertices i.e. image pixels are returned.

\subsection{Power ratio cut}

One of the major issues with spectral clustering is computational complexity. A ratio cut requires $\mathcal{O}\left(|V|^{\frac{3}{2}}\right)$ computations where $V$ is the set of vertices of the graph. Intuitively, it is expected that pairs of vertices with 'high' similarity should belong to the same component. This can be done by collapsing high similarity vertices together to hybrid vertices thereby reducing the size of the graph (see Fig. 16 for an illustration). However, two questions need to be answered: (1) how to quantify a 'high' similarity edge? (2) what are appropriate weights for the edges incident on the hybrid vertices so that spectral clustering on the collapsed graph yields similar results as that of spectral clustering on the original graph? PW framework answers these questions.

Applying the PW framework to Eq. 27, one can obtain the following collection of minimization problems labelled with $p$, where $p \in \mathbb{N}$ :

$$
\text { Minimize } \sum_{i} w_{i}^{p} \operatorname{Tr}\left(H^{T} L_{i} H\right) \text { subject to } H^{T} H=I_{k} \text {, }
$$

where the summation is over a distinct set of weights in the graph, $L_{i}$ is the Laplacian of the subgraph induced by the edges of weight $w_{i}$ (see Fig. 17 for an example) resetting the weights to 1 . It was shown in [12] that limit of minimizers to Eq. 28 as $p \rightarrow \infty$, also known as power ratio cut, can be computed as follows: The edges of the image graph are sorted in decreasing order of weights say $w_{l} \geq \cdots \geq w_{1}$. A graph is constructed with edges of highest weights $w_{l}$ of the image graph $\mathcal{G}$, i.e. the induced subgraph $\mathcal{G}_{>w_{l}}$. Edges are added to this graph gradually in decreasing order of weights. Then a critical value, i.e. $1<r \leq l$ is found such that $\mathcal{G}_{\geq w_{r}}$, the induced subgraph generated with edges of weights greater than or equal to $w_{r}$, has number of connected components greater than or equal to $k$ but $\mathcal{G}_{\geq w_{r-1}}$ has less than $k$ components. A spectral clustering is performed on $\mathcal{G}_{\geq w_{r-1}}$ subject to the condition that the representations on each of the connected components of $\mathcal{G}_{\geq w_{r}}$ are same. See Algorithm 4 from [12] for a formal algorithm.

Observe that the collapsed graph on which spectral clustering is performed is of much smaller size when compared to the original graph as $k \ll|V|$. Hence, the computation cost for the spectral clustering part is negligible in PW approximation. However, there is a sorting step involved and hence this algorithm runs in $\mathcal{O}(|V| \log |V|)$. This is a significant improvement as the classic ratio cut runs in $\mathcal{O}\left(|V|^{\frac{3}{2}}\right)$. Figure 18 from [12] 


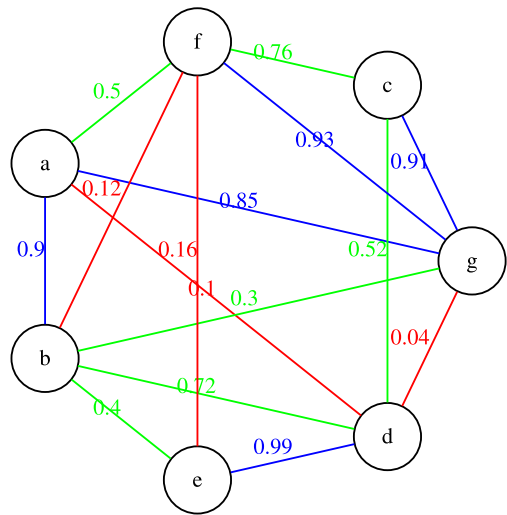

Fig. 14 Left: A similarity-based edge-weighted graph with seven vertices. The edge weights are displayed on the edges and are colour-coded to indicate the strength of similarity. Red, green, and blue indicate low, medium, and high, respectively. Right: 2 dimensional spectral embedding of the vertices obtained by solving the continuous relaxation of

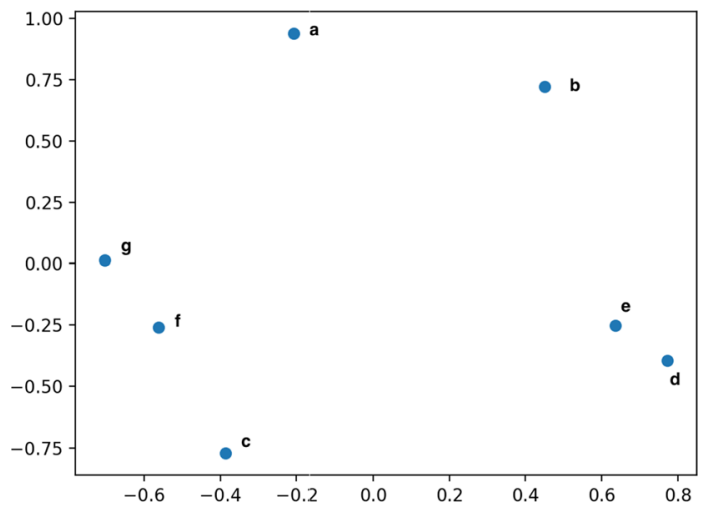

ratio cut. Observe that most of the pairs of vertices with higher similarity are closer and the pairs with low similarity are farther in the embedded space. Hence, a simple distance-based clustering would work well on the embeddings for clustering purposes
Fig. 15 Left: A simulated astronomical image to be segmented (same as left image in Fig. 8). Right: Clusters obtained by performing a ratio cut on the image on left. The details of implementation are provided in the text
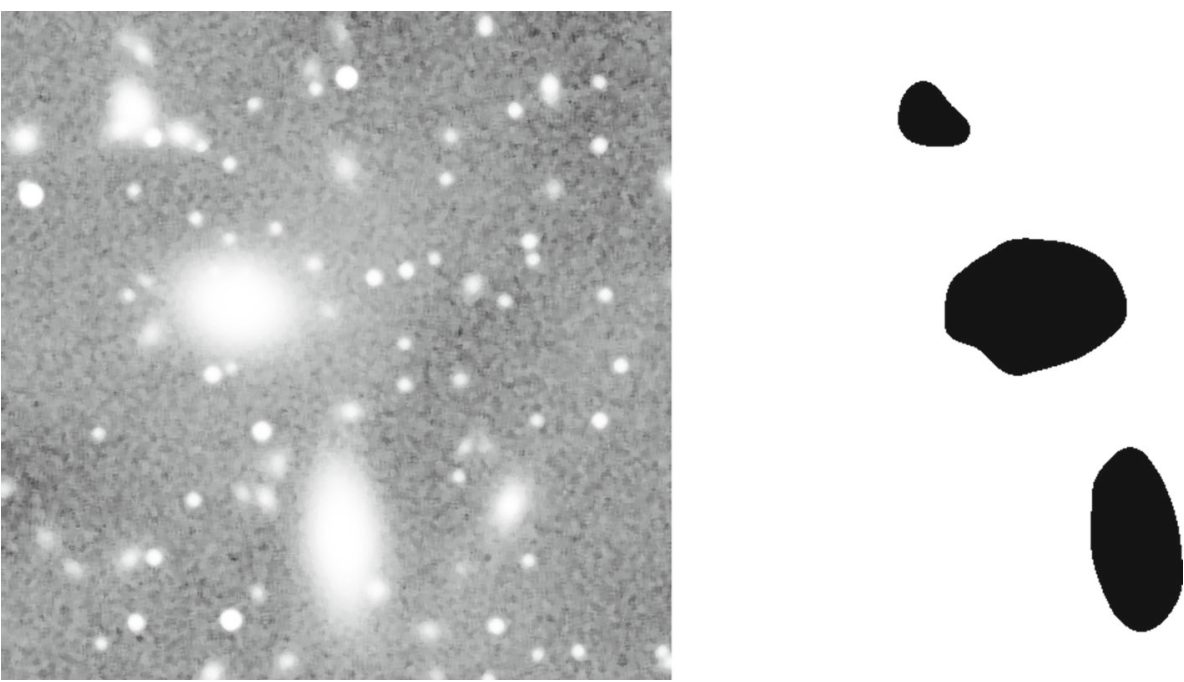
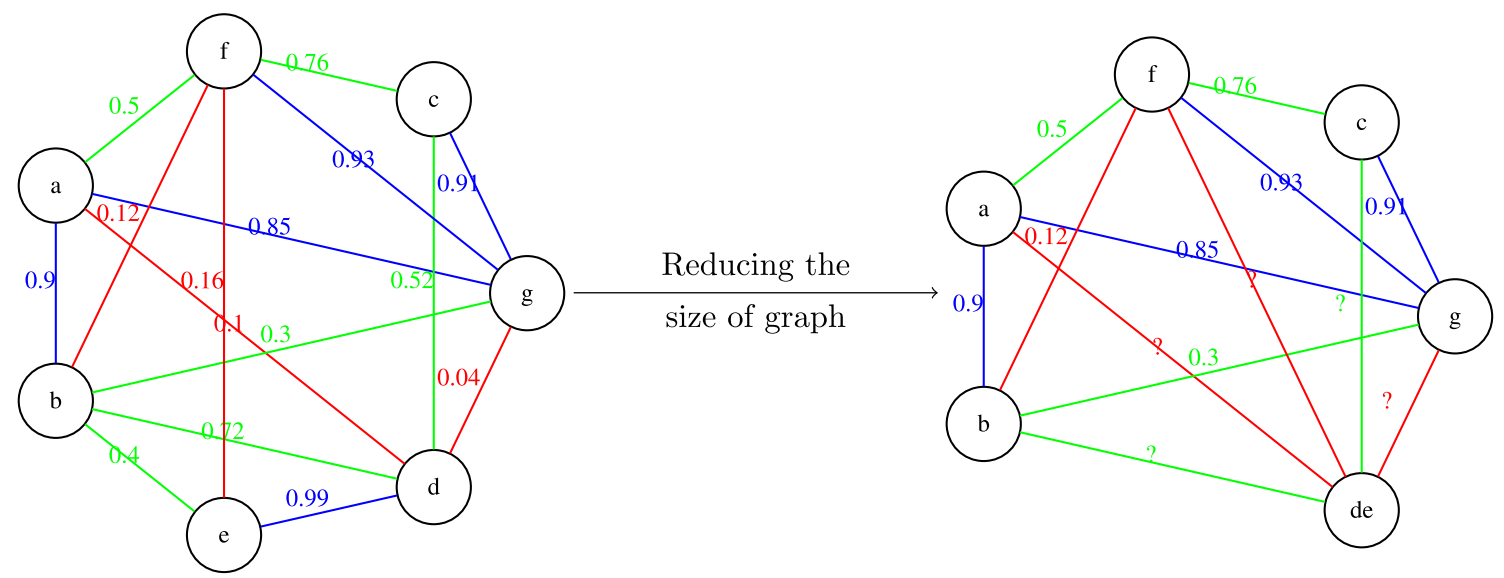

Fig. 16 Left: A similarity-based edge-weighted graph with seven vertices (this figure is replicated from [12]). The edge weights are displayed on the edges and are colour-coded to indicate the strength of similarity. Red, green, and blue indicate low, medium, and high respectively. Right: The vertices $d$ and $e$ are merged to form hybrid vertex $d e$. This hybrid vertex is adjacent to every vertex that either of $d$ and $e$ are adjacent. However, it is not clear on how the edge weights for the edges containing hybrid vertices have to be set so that ratio cut on this collapsed graph yields similar results as that of the original graph. PW answers this question 
Fig. 17 Top Left: A similarity-based edge-weighted graph with six vertices. The edge weights are displayed on the edges. Top Right, Bottom Left and Bottom Right: The edge-weighted graph is decomposed into several subgraphs, each consisting of edge weights of a fixed value in decreasing order of weights. These are called subgraphs induced by edges of fixed weight of the graph on top left
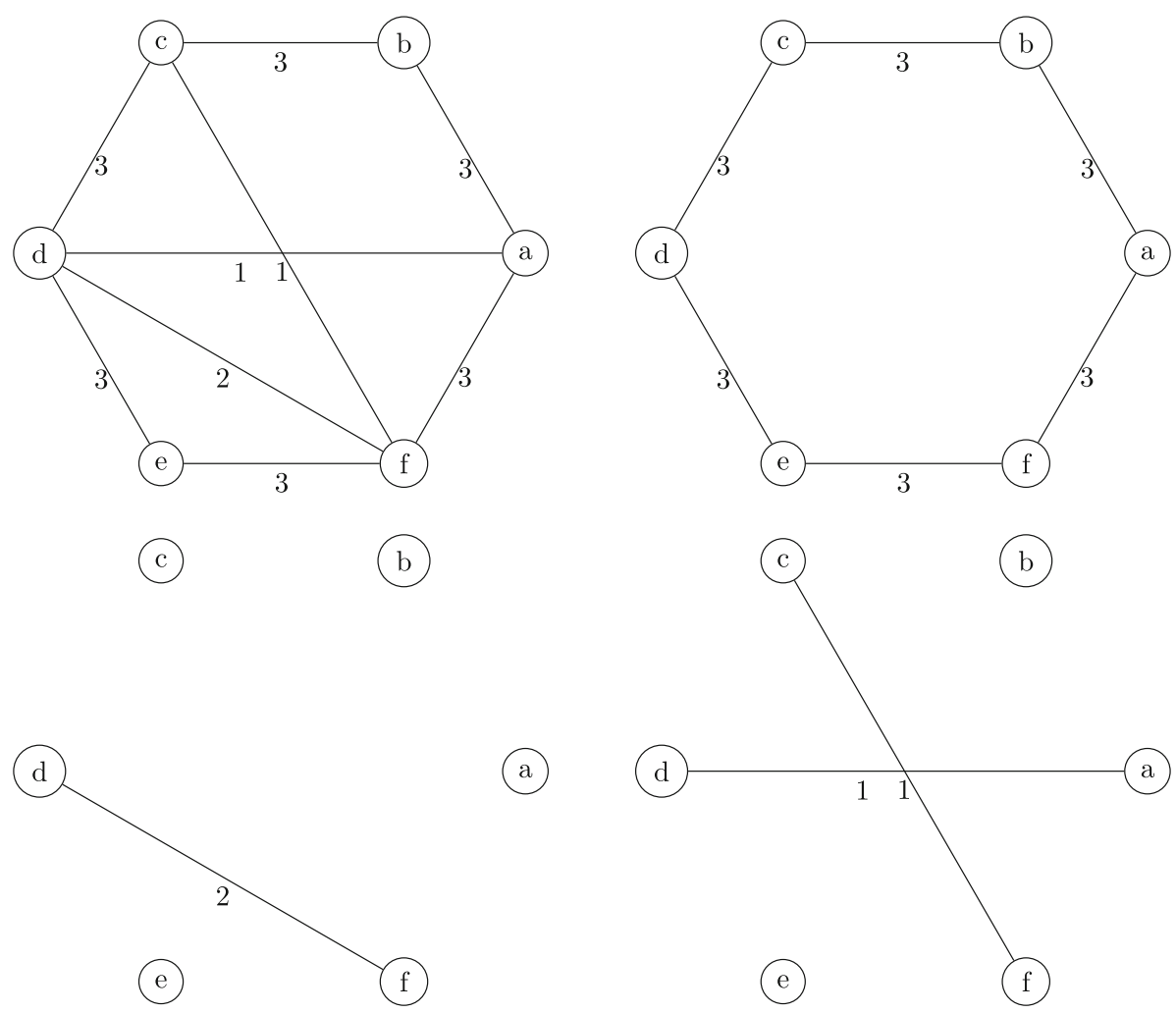

\section{$\overline{\text { Algorithm } 4 \text { PW Approximation to Ratio Cut Parti- }}$} tioning [12]

Input An edge-weighted graph $\mathcal{G}=(V, E, W)$ with bucketed weights $w_{1}<\cdots<w_{l}$

Output A representation of the subspace spanned by the PW approximation to ratio cut optimal solution.

1: Set $i=l$

2: while Number of connected components of $\mathcal{G}_{\geq w_{i}}$ is greater than or equal to $k$ do

3: $\quad$ Set $i=i-1$.

4: Let $\left\{C_{i}\right\}, i \in\left\{1, \ldots, n_{c}\right\}$ be the connected components in $\mathcal{G}_{\geq w_{i}}$.

5: Let $\bar{I}_{C_{i}}$ be the vector

$$
I_{C_{i}}(x)= \begin{cases}1 / \sqrt{\left|C_{i}\right|} & \text { if } x \in C_{i} \\ 0 & \text { otherwise }\end{cases}
$$

6: Construct matrix $N$ with $I_{C_{i}}$ as column vectors

7: Let $\mathcal{G}_{1}$ be the graph with vertex set same as that of $\mathcal{G}$.

Let $L_{1}$ be the unnormalized Laplacian of $\mathcal{G}_{1}$.

8: Let $\bar{L}_{1}=N^{t} L_{1} N$.

9: Calculate the first $k$ eigenvectors of $\bar{L}_{1}$ and construct $A$ using these eigenvectors as columns.

10: Return $N A$

shows the comparison of time taken to cluster simple blobs dataset [33] with $n_{\text {features }}=2$ and centers $=2$. Notice that as the number of data points increase, the PW approximation to ratio cut is a better option in terms of computations.

Also, it was shown in [10-12] that power ratio cut yields segmentation results comparable to that of the

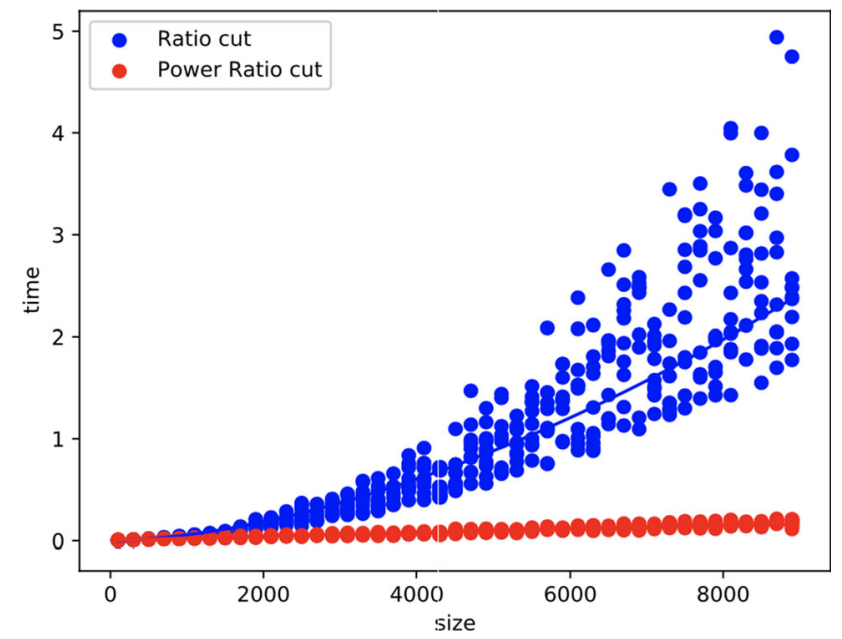

Fig. 18 A comparison of time complexities of PW approximation to ratio cut and ratio cut as a function of data size on blobs dataset [33] with parameters $-n_{\text {features }}=2$ and centers $=2$ (this figure is replicated from [12]). Observe that for small data sizes the difference between PW approximation and ratio cut is not significant, while for relatively larger data sizes, the difference is significant

ratio cut. An intuitive explanation on why the approximation is good is that the number of objects are much lesser in an image when compared to the number of pixels in the image. Hence, expensive computations (such as spectral clustering steps) are required only near the object boundaries and a simple greedy algorithm such as a maximum spanning tree-based label propagation 
suffices in the object interiors. In particular, for astronomical image segmentation, power ratio cut is a practical solution to implementing an approximation to the NP-hard ratio cut minimization as these images usually have a large number of pixels.

\section{Mutex watershed and power watershed optimization}

In the previous sections, we have seen random walker, spectral clustering and isoperimetric partitioning. Observe that for these methods to work well, a good estimate of the number of objects in the image should be computed beforehand. In the case of random walker, at least one seed pixel should be provided. Isoperimetric partitioning has to be applied recursively as many times as the number of objects in the image. Spectral clustering uses the number of objects as the input $k$ and partitions the graph into $k$ components.

In practical applications, it is often the case that the number of segments in an image are unknown and difficult to obtain a reasonable estimate. In such cases, one needs to apply algorithms that can yield good quality results which do not require the number of segments as an input. In this section, one such algorithm namely multi-cut problem and its PW approximation $[54,56]$ are reviewed in detail.

\subsection{Multi-cut: NP-hard problem}

Recall from Sect. 2, in some application areas, one can obtain information on certain pairs of pixels that are known to have the same labels and certain pairs to have different labels, each with a varied level of confidence. Hybrid image graphs capturing similarities and dissimilarities between pixels are used for such applications. One can then generalize the notion of graph-cut as follows: find a set of edges, possibly both similarity and dissimilarity edges (called cut edges) such that sum of their weights is minimum. Here the segmentation is obtained by discarding the dissimilarity edges after the removal of the cut edges from the image graph. One expects that the endpoints of the discarded dissimilarity edges belong to different segments. Hence, there is a requirement of an additional condition on the selected cut edges which is: removal of the cut edges should not result in a cycle with exactly one dissimilarity edge (see Fig. 19 for an illustration on violation of this condition).

Observe that the sum of weights of all edges in the image graph is constant. Hence, minimizing the sum of weights of cut edges is the same as minimizing the negative-sum of weights of edges that do not belong to the cut. Denote the set of edges that do not belong to the cut as $A$, the multi-cut problem can be stated as the following optimization problem:

$$
\begin{gathered}
\text { Minimize } Q(\mathbf{a})=-\sum_{e \in E} a_{e} w_{e} \\
\text { subject to } \mathbf{a} \in\{0,1\}^{|E|}, \mathcal{C}_{1}(A)=\emptyset \\
\text { with } A=\left\{e \in E \mid a_{e}=1\right\},
\end{gathered}
$$

where $E$ denotes the set of edges of the image graph, $\mathcal{C}_{1}(A)$ denotes the set of cycles in $A$ with exactly one dissimilarity edge. Unfortunately, Eq. 30 is a NP-hard problem.

\subsection{Interpreting mutex watershed as power watershed approximation to multi-cut}

In $[54,56]$, the authors propose an approximation solution to Eq. 30 with a greedy algorithm. This algorithm combined with a CNN architecture to learn the edge weights achieved state-of-the-art results on EM segmentation challenge [5] when published. It was shown that this algorithm can be interpreted as an application of PW on the NP-hard multi-cut problem.

$$
\begin{aligned}
& \text { Minimize } Q^{(p)}(\mathbf{a})=-\sum_{e \in E} a_{e} w_{e}^{p} \\
& \text { subject to } \mathbf{a} \in\{0,1\}^{|E|}, \mathcal{C}_{1}(A)=\emptyset \\
& \text { with } A=\left\{e \in E \mid a_{e}=1\right\} .
\end{aligned}
$$

Decomposing the edges of the graph into different weights in decreasing order of magnitudes $w_{l}>\cdots>$ $w_{1}$ (see Fig 17), i.e. $E_{r}=\left\{e \in E \mid w_{e}=w_{r}\right\}$ for each $1 \leq r \leq l$. The first level in the PW nested minimization problem is given by

$$
\begin{aligned}
& \text { Minimize }-\sum_{e \in E_{l}} a_{e} \\
& \text { subject to } \mathbf{a} \in\{0,1\}^{\left|E_{l}\right|}, \mathcal{C}_{1}(A)=\emptyset \\
& \text { with } A=\left\{e \in E_{l} \mid a_{e}=1\right\} .
\end{aligned}
$$

Let the solution space be denoted by $A_{l}$.

Minimizing Eq. 32 is no longer NP-hard as all the weights in the subgraph are of the same weight. One can then greedily add edges so as to satisfy the cycle condition [15]. At the next level, we have the subgraph with edges $E_{l-1}$ :

$$
\begin{gathered}
\text { Minimize }-\sum_{e \in E_{l-1}} a_{e} \\
\text { subject to } \mathbf{a} \in\{0,1\}^{\left|E_{l-1}\right|}, \mathcal{C}_{1}(A)=\emptyset \\
\text { with } A=A_{l} \cup\left\{e \in E_{l-1} \mid a_{e}=1\right\} .
\end{gathered}
$$

Let the solution space be denoted by $A_{l-1}$. This process is continued all the way until edges are exhausted, i.e. $E_{1}$. The solution obtained is $A_{1}$. Discarding the dissimilarity edges from $A_{1}$ yields a segmentation satisfying the cycle constraint. See Algorithm 5 from [54] for a formal algorithm. Here $\mathcal{C}_{0}(A)$ denotes the set of cycles 


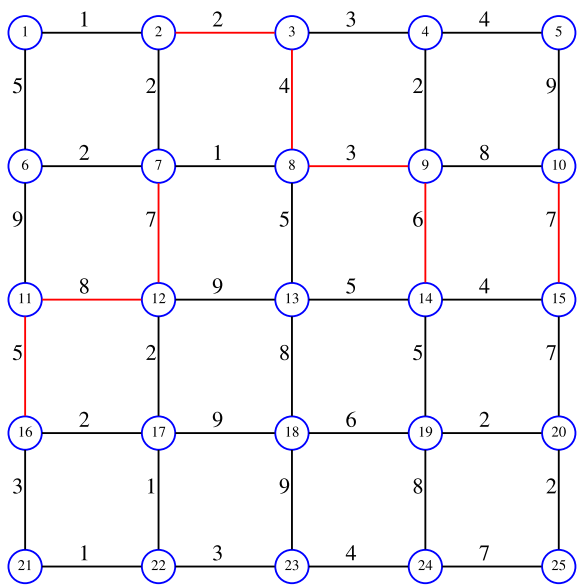

Fig. 19 Left: A hybrid graph on a toy image capturing similarities and dissimilarities between the pixels. Each vertex represents a pixel. Red coloured edges indicate dissimilarity and black coloured edges indicate similarity. The weights on the corresponding edges indicate the strength of similarity/dissimilarity. Right: A set of cut edges removed

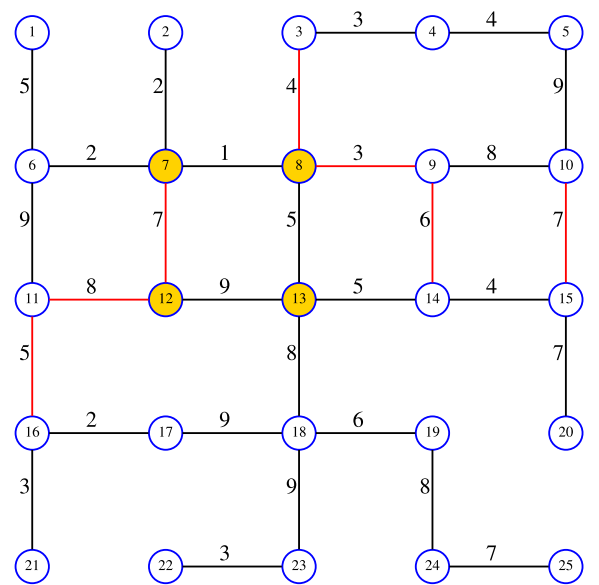

on the image graph on left. Observe that these cut edges violate a desired condition. The cycle $\langle 7,8,13,12\rangle$ consisting of edges , $\{7,8\},\{8,13\},\{13,12\},\{12,7\}$ have exactly one dissimilarity edge namely $\{12,7\}$. Removal of the edge $\{12,7\}$ would still lead to pixels 12 and 7 to contain in the same segment
Fig. 20 Left: The set of edges selected by mutex watershed when applied on Fig. 19 left image. Observe that there are no cycles with exactly one red edge. Hence there is no ambiguity in the partitions. Right: The final partition obtained by mutex watershed. This is obtained by discarding the red edges in the left figure
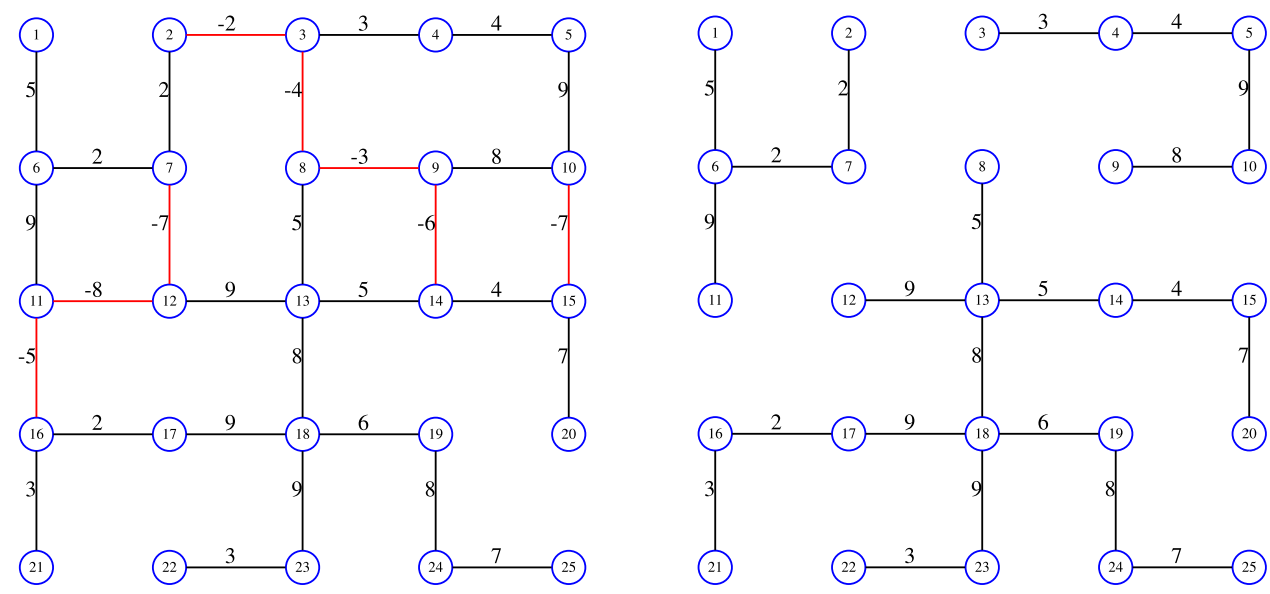

in $A$ with no dissimilarity edges. The result obtained by applying mutex watershed on Fig. 19 left image is illustrated in Fig. 20. Figure 21 replicated from $[54,56]$ shows an image from EM segmentation challenge [5] and illustrates how mutex watershed works on a real image.
To summarise, application of PW to multi-cut allows one to practically implement a multi-cut minimization and obtain high quality image segmentation results. In case of astronomical images, learning appropriate edge weights (using a neural network architecture) suitable for mutex watershed algorithm is a potentially useful research direction.

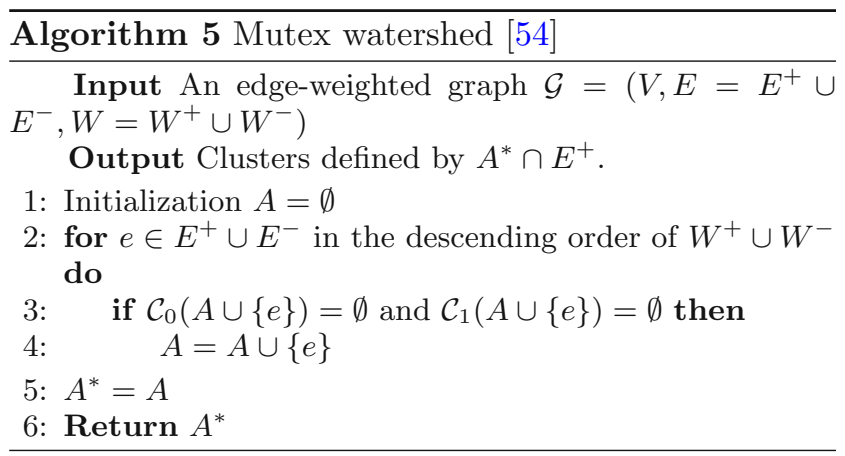

\section{Explaining the links between spanning tree filters and shortest path filters using PW}

Recall from Sect. 1 that image filtering is the process of summarizing the image by removing redundant details. The relevant information and the redundant details depend on the application at hand. A popular class of filters are edge-preserving image filters i.e. an operation on the image that blurs the details within objects and preserves the object boundaries. The bilateral filter 

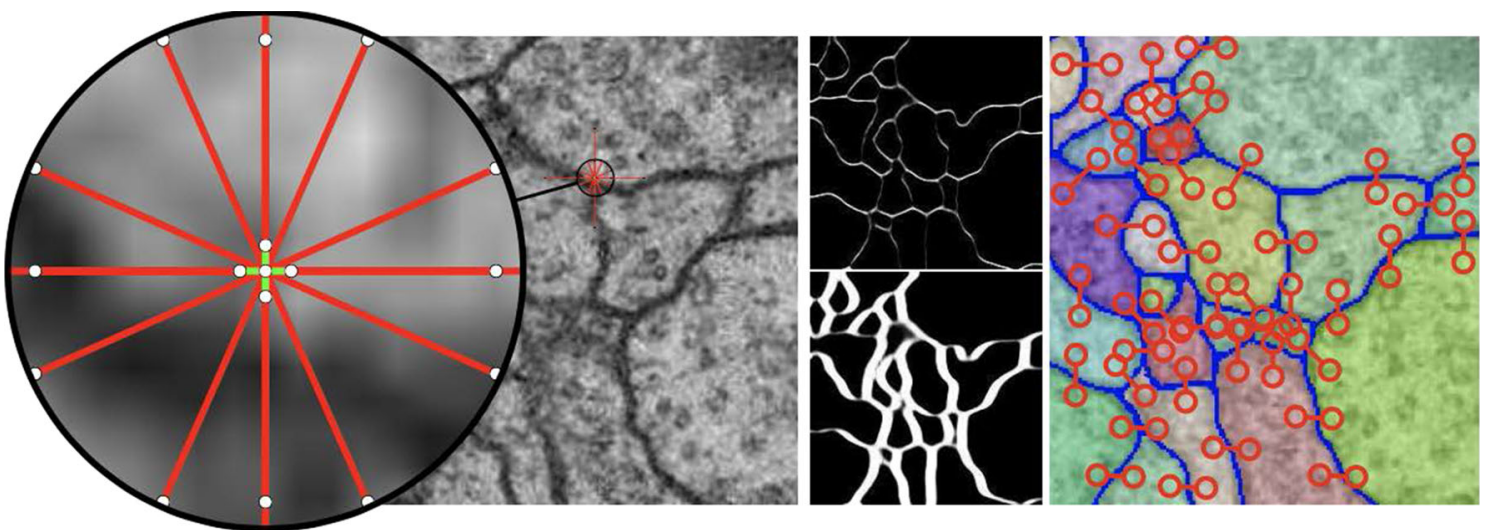

Fig. 21 This figure is replicated from $[54,56]$. Left: Overlay of raw data from the ISBI 2012 EM segmentation challenge and the edges for which attractive (green) or repulsive (red) interactions are estimated for each pixel using a CNN. Middle: vertical/horizontal repulsive interactions at

illustrated in Fig. 2 is an example of an edge-preserving filter. There is a vast amount of literature on edgepreserving filters.

In this section, we restrict the discussion to two families of graph-based weighted average filters namely shortest path filters and spanning tree based filters. As the name suggests, graph-based filters implies that the filtering is performed using a graph model on the image. The filtered value at each pixel is given by a weighted average of the other pixels in the image. The pairwise weights in the shortest path filters arise from a shortest path between pairs of pixels. On the other hand, the pairwise weights in the spanning tree-based filters are computed from spanning trees.

\subsection{Shortest path filters}

Recall from Sect. 2 that dissimilarity based edgeweighted graphs are used for image filtering. The family of the shortest path filters can be described as follows. The filtered value at pixel $i$ is given by

$$
\mathrm{SPF}_{i}=\sum_{j \in V} g_{i}(j) I_{j}
$$

where $g_{i}(j)=\frac{\exp \left(-\frac{\Theta(i, j)}{\sigma}\right)}{\sum_{q \in V} \exp \left(-\frac{\Theta(i, q)}{\sigma}\right)} \cdot \Theta(i, j)$ is the smallest number of edges on paths among all shortest path between pixels $i$ and $j$ on the image graph. $\sigma$ is a smoothing parameter. Shortest paths can be defined in many ways $[13,24,35]$. Figure 22 illustrates a standard definition of a shortest path distance. Intuitively, pixels which are in the same object are separated by shorter paths when compared to pixels across objects. Thus, it is expected that such a filter would result in smoothing of the image keeping the object boundaries intact.

Although, shortest path filters are theoretically promising, they are computationally expensive. This is because an exact computation [25] requires finding shortest intermediate/long range are shown in the top/bottom half. Right: Active mutual exclusion (mutex) constraints that the proposed algorithm invokes during the segmentation process

paths between all pairs of vertices in the graph which is $\mathcal{O}\left(|V|^{3}\right)$ where $|V|$ is the number of vertices of the image graph.

\subsection{Spanning tree filters}

On the other hand, spanning tree based filters were introduced independently w.r.t. shortest path filters. The spanning tree filters are weighted average filters. The pairwise similarity weights are computed on an arbitrary minimum spanning tree (MinST) on the image graph. A specific spanning tree filter namely Tree filter [6] can be described as follows. The filtered value at pixel $i$ is given by

$$
\mathrm{TF}_{i}=\sum_{j} t_{i}(j) I_{j}
$$

where $t_{i}(j)=\frac{\exp \left(-\frac{D(i, j)}{\sigma}\right)}{\sum_{q} \exp \left(-\frac{D(i, q)}{\sigma}\right)} . D(i, j)$ is the number of edges on an arbitrary MinST of the graph (this quantity is well defined as given an arbitrary spanning tree, there exists a unique path between every pair of vertices in the graph). $\sigma$ is a smoothing parameter. An illustration of the spanning tree filter on a toy example is provided in Fig. 22. Figure 23 provides an illustration of the tree filter on a simulated astronomical image. In general, for pairs of pixels $i$ and $j$ belonging to different objects, $D(i, j)$ is large. However, there is at least one boundary edge in any arbitrary MinST. In practice such edges are usually negligible in number. Thus, tree filter works reasonably well in practice except for a small leak at object boundaries. A tree filter can be computed efficiently in linear time [58] (using two passes, bottom-up and top-down).

In $[21,23]$, it was shown using PW framework that the tree filter is a fast approximation to the contrastinvariance version of a shortest path filter. The exact contrast invariant version of a shortest path filter was 

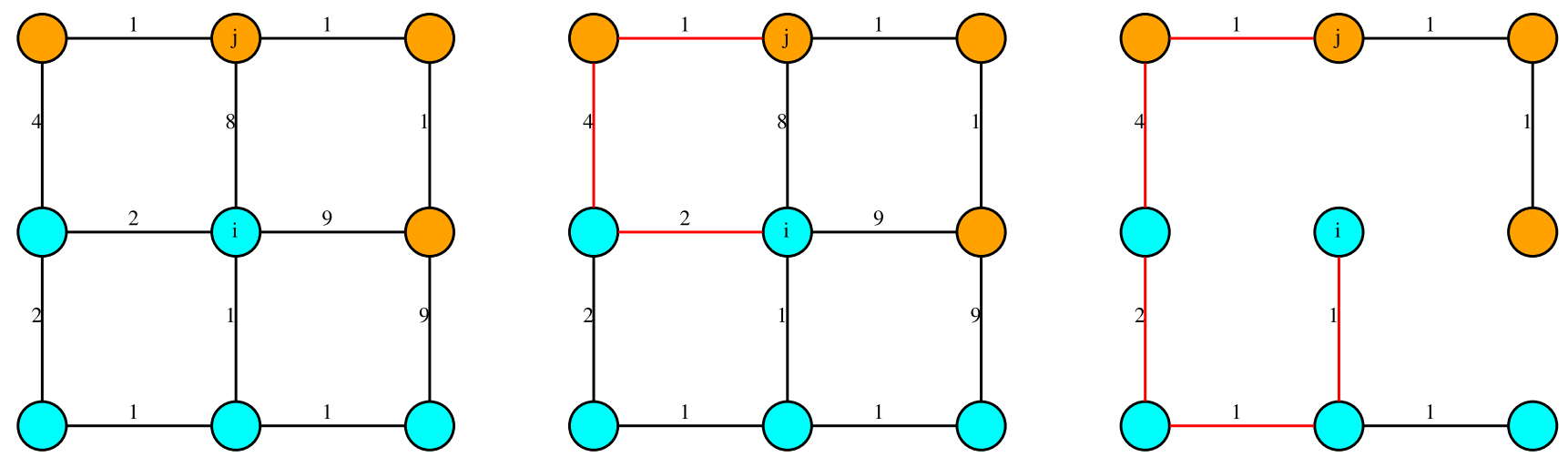

Fig. 22 Left: A toy image represented with an edgeweighted graph. Vertices represent pixels and the edge weights represent dissimilarity between adjacent vertices. The image contains two objects and the pixels corresponding to different objects are highlighted in different colours. Middle: The shortest path between the pixels $i$ and $j$ is highlighted in red. Here, a shortest path is determined by the sum of the weights of its edges. Observe that every other path between $i$ and $j$ has sum of the edge weights strictly greater than that of the path highlighted in red. Thus $\Theta(i, j)=3$. Right: The unique path between the pixels $i$ and $j$ is highlighted in red, i.e. $D(i, j)=5$. Notice that for many pairs of pixels across objects, i.e. pairs such that both pixels belonging to different objects, the separation on the MinST is larger than that of their spatial distance
Fig. 23 Top left: A simulated astronomical image to be segmented (same as left image in Fig. 8). Top right: A Canny edge detector applied on the image on top left. Bottom left: A tree filter is implemented on the image on left using Eq. 35.

Bottom right: A Canny edge detector applied on the tree filtered image. The boundaries identified by a simple edge detector such as Canny edge detector are more reliable on the tree filtered image
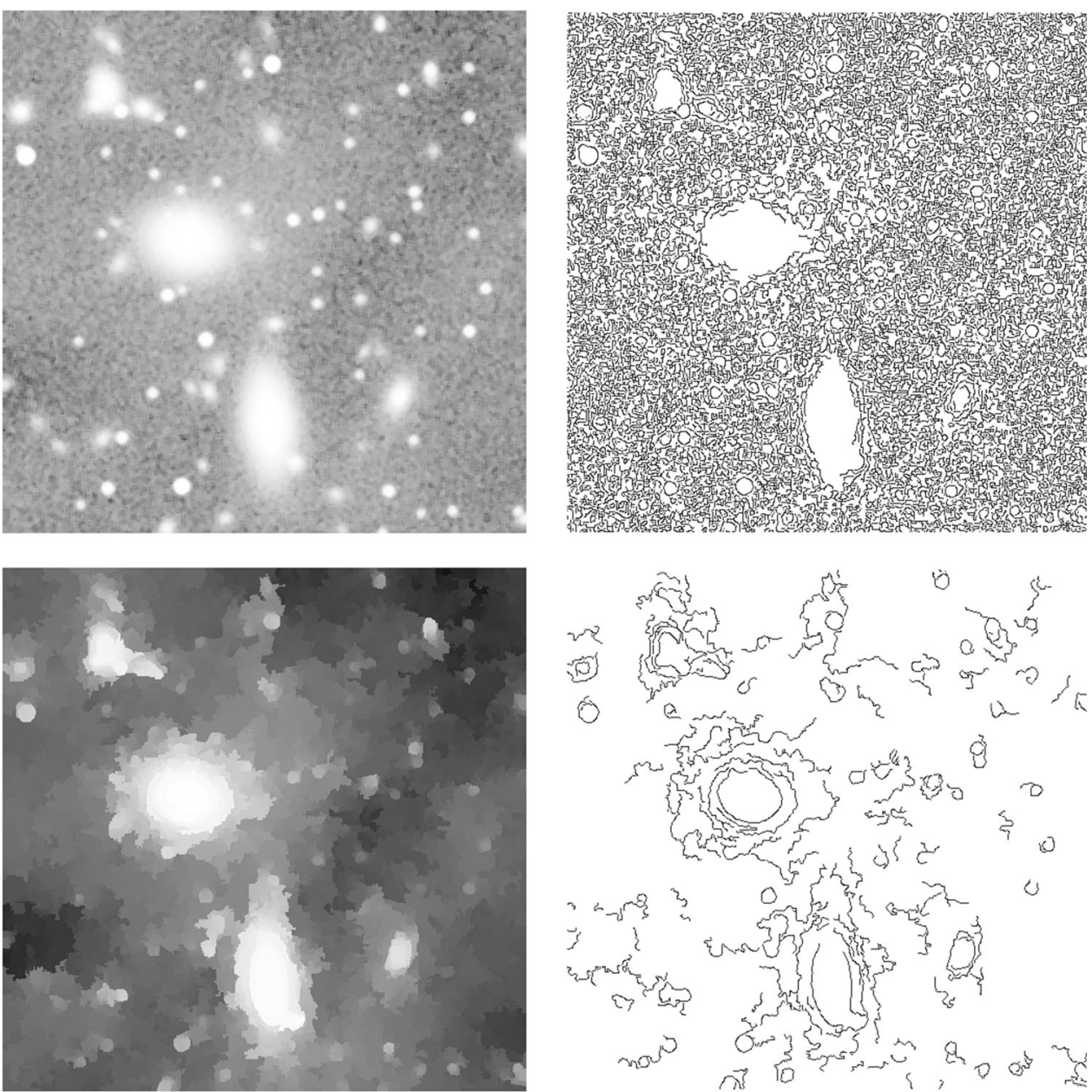
characterized as a weighted average filter with the pairwise weights computed on UMinST of the image graph. Recall that UMinST of a graph is a subgraph generated by edges of all MinST's of the graph. Two other approximations to obtain the contrast invariant version of a shortest path filter, namely order-based approximation and depth-based approximation have been proposed in [23]. Algorithms 6, 7, and 8 provide details on implementation of these approximations. The key idea to implementing these approximations is that for every pixel in the image, there exists a spanning tree from which the pairwise weights for filtering this pixel can be computed. Such a tree is termed as an adaptive spanning tree in [23]. These adaptive spanning trees can be computed in parallel to obtain these approximations more efficiently. Further, it was shown empirically that the tree filter and these approximations yield similar results in practice.
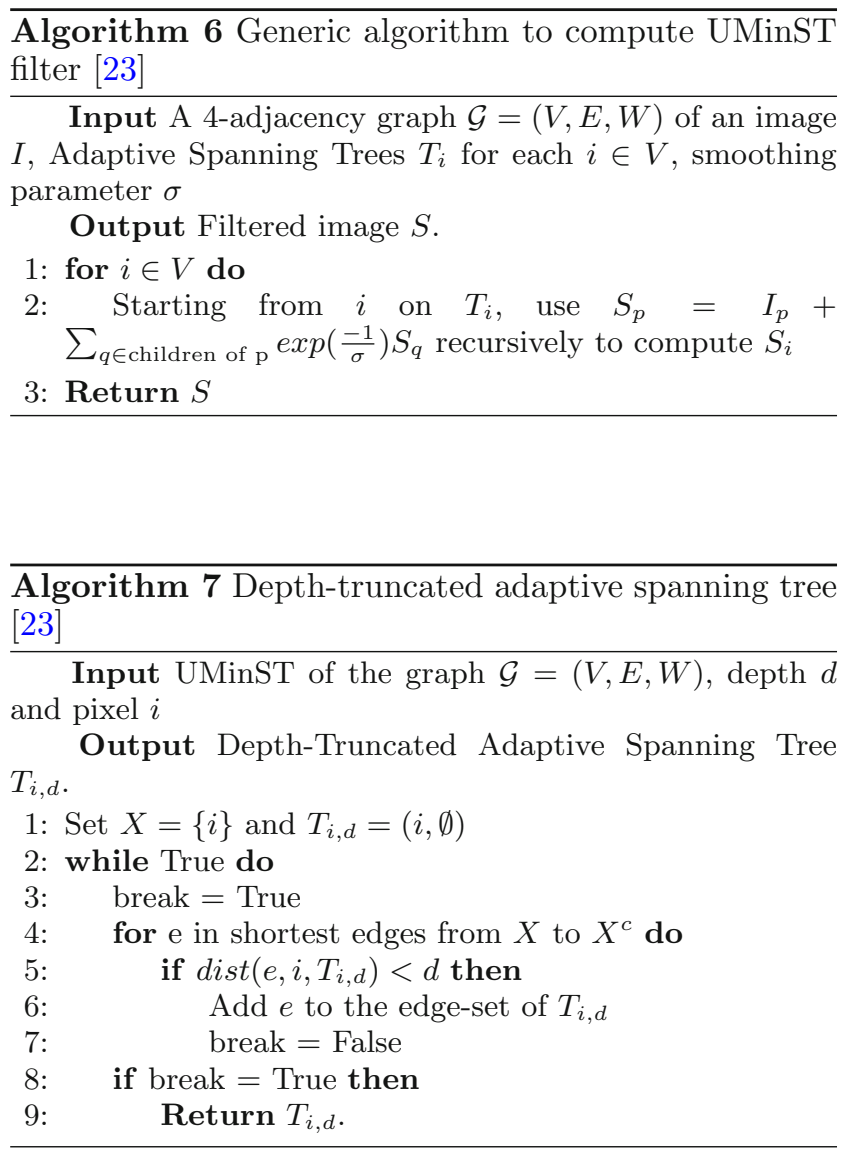

\section{Experiments on simulated astronomical sky images}

In this section, experiments are performed on simulated astronomical sky images. It is a common practice to use simulations $[31,40,47,52]$ as it is difficult to

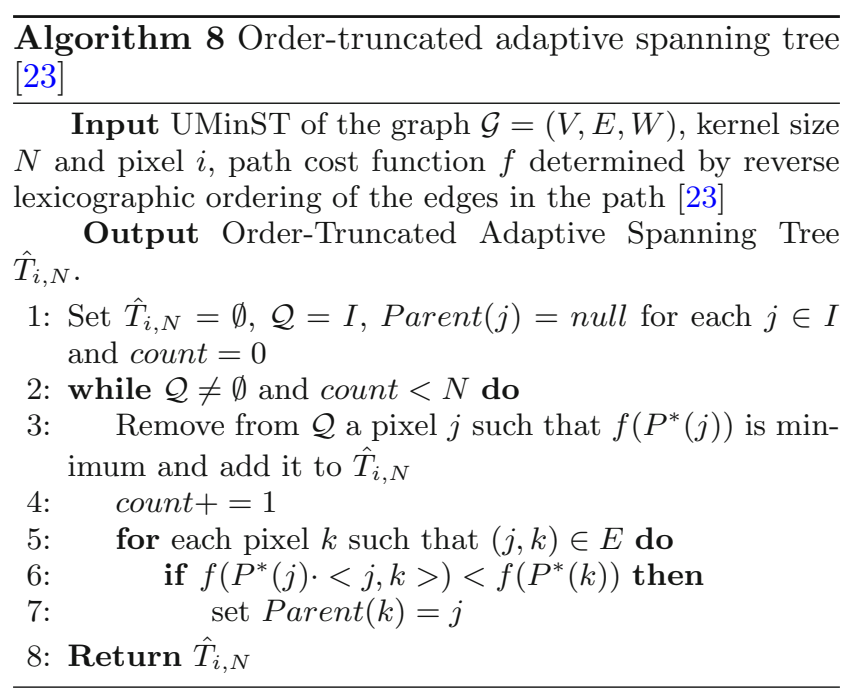

obtain ground truth segmentation for real astronomical images. The sky images are generated using the $\mathrm{R}$ code developed by authors in [43]. First, noise-free sky images with light sources are generated. A threshold is then applied on the noise-free simulations to obtain a ground truth foreground and background. Typical noise such as Viking object shot-noise, sky noise and Pareto noise are then added to simulate real sky images. See Fig. 24 for an illustration. The noisy images are used for segmentation and filtering.

The goal of segmentation is to separate the foreground from the background, i.e. separate the background sky from the light sources. Recall that one of the the aims of the article is to demonstrate that the implementation of classic graph-based cost minimization methods and the corresponding $\mathrm{PW}$ versions yield similar results. F-ratio and AUC curves are used evaluating segmentation and filtering results. As the tutorial article does not attempt to achieve state-ofthe-art results, sophisticated measures such as pairsof-pixel method [42] or tailor-made evaluation measures for astronomical sky images such as described in [31] are not used. The second aim of the article is to show that the PW versions scale well when compared to the classic implementations. This is demonstrated experimentally by implementing both the algorithms on a same machine (Intel(R) Xeon(R) CPU E5620 at $2.40 \mathrm{GHz}$ with RAM size of 16 GigaBytes) and comparing the computation times. All the segmentation methods described in the article are similar to implement. Hence, segmentation experiments are performed comparing only one segmentation method, namely isoperimetric partitioning, i.e. the classic implementation of isoperimetric partitioning versus the corresponding $\mathrm{PW}$ version. Also, these segmentation methods yield hard labels. Hence, F-ratio is used to evaluate the quality of the segmentation. The higher the F-ratio, the better the segmentation results.

The isoperimetric partitioning is performed as follows: the noisy images are pre-processed with a threshold operation followed by a morphological opening [45]. 


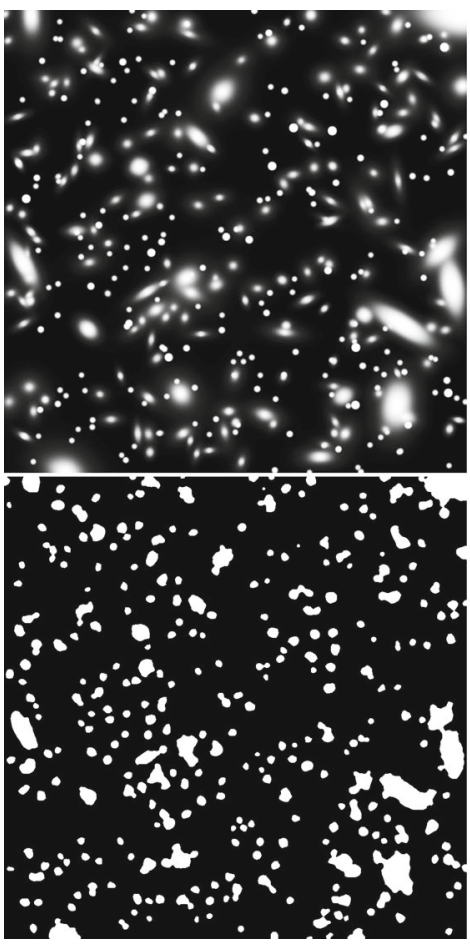

Fig. 24 Top left: A simulation of a noise-free astronomical sky image. Top middle: A ground truth of foreground and background obtained by thresholding the image on left. Top right: Viking object shot-noise, sky noise and Pareto noise added to the noise-free image to simulate a real astronomical sky image [43]. A median filtered version of the noisy image is illustrated for better visualization. Bottom: All three segmentations are obtained on the raw noisy image as described in the text. Bottom left: Result of segmentation obtained by
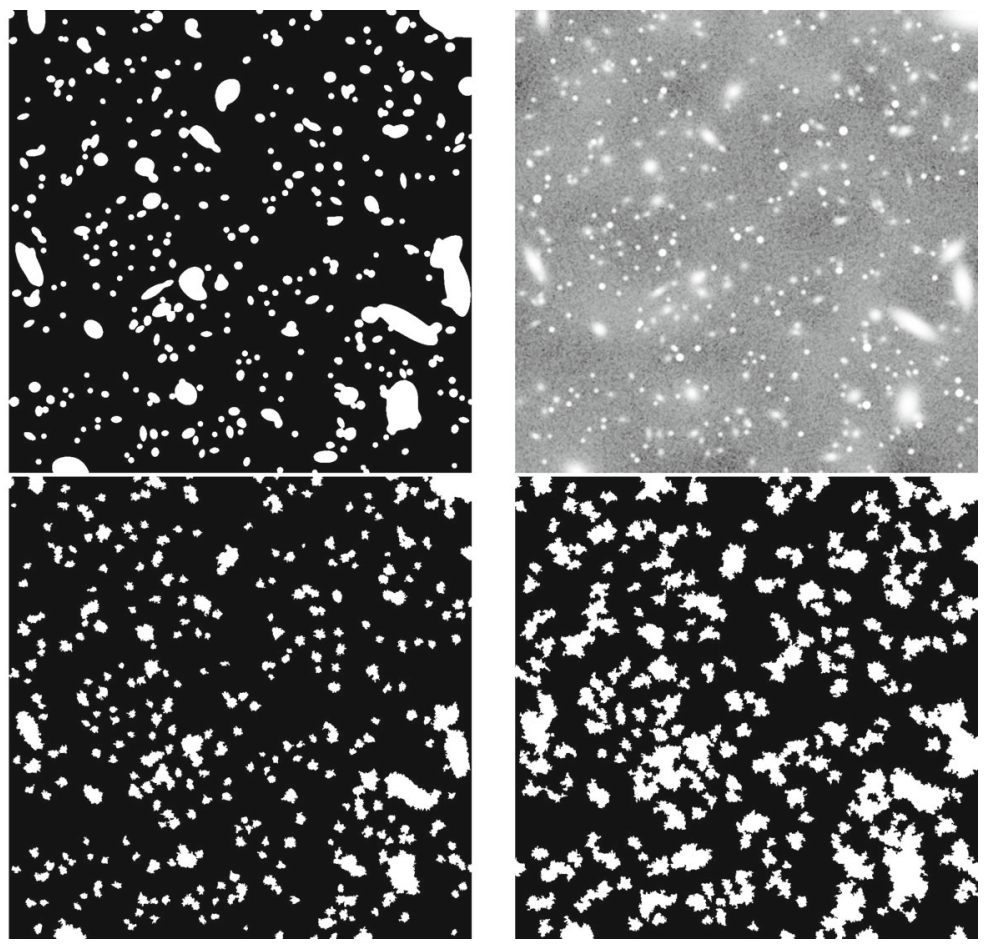

implementation of classic approach to isoperimetric segmentation (see Eq. 21). Bottom middle: PW implementation of isoperimetric segmentation (i.e. solving Eq. 22 on UMaxST) yields similar results to that of the classic approach. Bottom Right: Result of solving Eq. 22 on an arbitrary MaxST, i.e. the method in [26]. Observe that qualitatively these results appear different when compared to the figure on bottom left. This observation is consistent with left figure in Fig. 12

tation of isoperimetric segmentation. Simulated astronomical images of 10 different sizes and 30 images of each size, starting from a smallest size of $100 \times 100$ to a largest size of $1000 \times 1000$ are used. The $l$ th size has width and height each of $100 l$, i.e. its number of pixels are $l^{2}$ times the number of pixels in the smallest size. The ratio of the mean computational time for each image size to the mean computational time of the smallest size are plotted on the $y$ axis. The values on the $x$ axis have to be interpreted as the ratio of number of pixels in the image to the number of pixels in a $100 \times 100$ image. For example, a value of 81 on the $x$ axis indicates that the image is of the shape $900 \times 900$ and has $81 \times 10^{4}$ pixels in total. It can be seen that PW counterpart of the isoperimetric segmentation scales well (the green plot) while the classic version (the blue plot) is not scalable.

For comparing the filtering methods, a bilateral filter is used to compare against the tree filter instead of a shortest path filter. This is because a bilateral filter is a widely used as an edge-preserving filter in practice. Also, shortest path filters are prohibitively slow to implement. The filters are compared as follows: a noisy image is filtered using both the filtering methods. As these filters are supposed to smooth pixel val-
Figure 25 compares the scalability of the PW-based isoperimetric segmentation with the classic implemen- 
Table $1 F$ ratio

\begin{tabular}{llll}
\hline & \multicolumn{2}{l}{ Mean $F 1$ ratio on 30 simulated images } \\
\cline { 2 - 4 } & Iso & PW Iso & MST Iso \\
\hline Mean F-ratio & 0.74 & 0.75 & 0.68 \\
Std. Dev. F-ratio & 0.07 & 0.06 & 0.08 \\
\hline
\end{tabular}

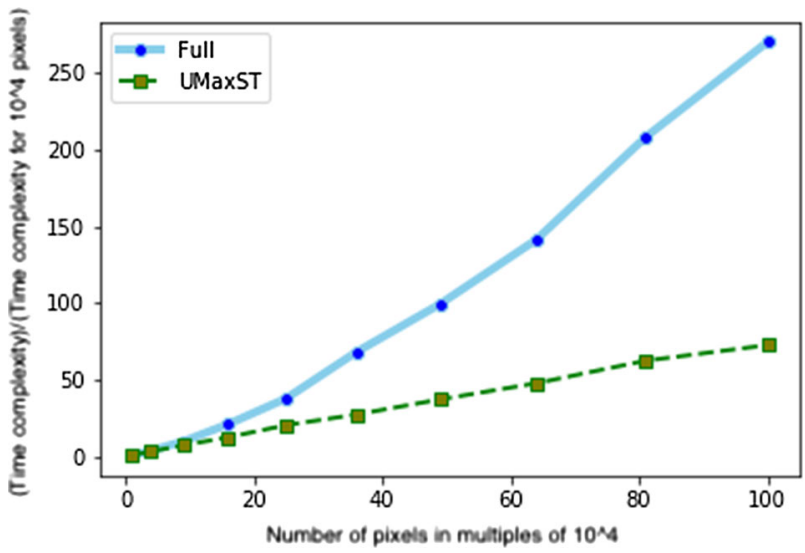

Fig. $25 X$-axis represents the number of pixels (in multiples of $10^{4}$ ) in the image on which segmentation is performed. A point $\left(x_{0}, y_{0}\right)$ has to be interpreted as follows: $y_{0}$ represents the ratio of mean time taken for implementation of a segmentation method on an image with $x_{0} \times 10^{4}$ pixels to the mean time taken to implement the same method on an image with $10^{4}$ pixels. The blue plot corresponds to the classic implementation of isoperimetric partitioning and the green plot corresponds to the PW version. It can be clearly seen that the PW version scales much better when compared to the classic implementation

ues within objects without losing the boundary details, simple thresholds on the filtered images are expected to achieve good segmentation results. The foregroundbackground segmentation problem as described earlier is used for this purpose. The filtered images are post-processed with an opening operator [46] and each threshold, the segmentation result is compared against the ground truth w.r.t. AUC. An average AUC measure over each threshold is used as a quality measure of the segmentation. A higher average AUC indicates a better quality segmentation and thus a better filtering approach. It can be seen from Table 2 that tree filter outperforms the classic bilateral filter used for edgepreserving filtering.

A tree filter, an approximation to the contrast invariance implementation of shortest path filter thus yields good filtering results. It is easy to see that the bottleneck of the tree filter is the computation of a MinST. This is because the filtered values are obtained on the tree in two passes and can be obtained in linear time. On the other hand, a MinST computation required a sorting step which is $\mathcal{O}(|V| \log |V|)$. Thus, tree filter scales better than a shortest path filter which has asymptotic complexity of $\mathcal{O}\left(|V|^{3}\right)$.

\section{Conclusions and perspectives}

In this article, several popular graph-based approaches to image segmentation and filtering, namely random walker segmentation, isoperimetric partitioning, ratio cut, multi-cut and shortest path edge-preserving filters are revisited. The applications of PW framework to these methods are surveyed and analysed from the perspective of contrast invariance. It is shown that the PW versions of these methods can be visualized as contrast-agnostic fast approximations to the corresponding methods. These methods fall under a large class of cost minimization problems on finite graphs. This class encompasses all cost functions that can be written as a weighted linear combination of pairwise penalties on pixel labels such that the weights are monotonic functions of the corresponding weights of the edges connecting the pixels. On this particular class of cost minimization problems, the PW framework operates on a specific substructure of the graph, i.e. either of UMinST or UMaxST. For graph-based cost minimization approaches to image processing with more general cost functions, the PW framework can still be applied by considering the sequence of nested minimization problems as mentioned in the article. This results in scalable algorithms and is potentially useful in image processing applications such as astronomy where images with massive number of pixels needs to be processed.

A recent trend in the usage of graph-based optimization in imaging applications is to learn the edge weights of the underlying image graph using deep neural networks. For example, an end-to-end learned random walker proposed in [9] achieved state-of-the-art results on some image segmentation database [20]. However, these methods do not scale well. PW is compatible with such end-to-end learned graph-based methods and can be applied at the test phase. This would be useful in building scalable state-of-the-art models for image segmentation and filtering. As a current instance of such research direction, the mutex watershed [54-56] was shown to achieve state-of-the-art results on a popular image segmentation database [5] and high-quality results on popular semantic segmentation databases $[14,38]$, using edge-weights obtained thanks to a CNN architecture. In case of astronomical images, learning appropriate edge weights (using a neural network architecture) suitable for mutex watershed algorithm is a potentially useful research direction. Essentially, domain knowledge of the physical characteristics of astronomical images can be incor- 
Table 2 AUC

\begin{tabular}{llr}
\hline & Mean AUC on 30 simulated images & TF \\
\cline { 2 - 3 } & BF & 0.79 \\
\hline Mean & 0.61 & 0.03 \\
Std. Dev. & 0.03 & \\
\hline
\end{tabular}

porated into the weights of edges. PW has also been used for other interesting applications such as surface reconstruction [16] and estimation of separating planes between touching 3D objects [34]. Anisotropic diffusion for L0 [17] is another interesting direction of research.

Acknowledgements Sravan Danda would like to acknowledge the funding received from BPGC/RIG/2020-21/112020/01 (Research Initiation Grant provided by BITSPilani K K Birla Goa Campus) and thank APPCAIR, and Computer Science and Information Systems, BITSPilani Goa. Aditya Challa would like to thank Indian Institute of Science (IISc) for the Raman Post Doctoral fellowship. The work of B. S. D. Sagar was supported by the DST-ITPAR-Phase-IV project under the Grant number INT/Italy/ITPAR-IV/Telecommunication/2018. Laurent Najman would like to acknowledge the funding received from - ANR-15-CE40-0006 CoMeDiC, ANR- 14-CE27-0001 GRAPHSIP research grants and Programme d'Investissements d'Avenir (LabEx BEZOUT ANR-10-LABX- 58). He would also like to thank the European Union's Horizon 2020 research and innovation program under the Marie Skłodowska-Curie grant agreement No. 721463 to the SUNDIAL ITN network.

\section{References}

1. C. Allène, J.-Y. Audibert, M. Couprie, J. Cousty, Renaud Keriven et al., Some links between min-cuts, optimal spanning forests and watersheds. ISMM 1, 253264 (2007)

2. Alpert, S., M. Galun, R. Basri, A. Brandt, Image segmentation by probabilistic bottom-up aggregation and cue integration. In: Proceedings of the IEEE Conference on computer vision and pattern recognition (2007). https://ieeexplore.ieee.org/abstract/document/ 4270042

3. P. Arbelaez, M. Maire, C. Fowlkes, J. Malik, Contour detection and hierarchical image segmentation. IEEE Trans. Pattern Anal. Mach. Intell. 33(5), 898-916 (2011)

4. P. Arbeláez, J. Pont-Tuset, J.T. Barron, F. Marques, J. Malik, Multiscale combinatorial grouping. In: Proceedings of the IEEE Conference on computer vision and pattern recognition, pp.328-335 (2014). https:// doi.org/10.1109/CVPR.2014.49

5. I. Arganda-Carreras, S.C. Turaga, D.R. Berger, D. Cireşan, A. Giusti, L.M. Gambardella, J. Schmidhuber, D. Laptev, S. Dwivedi, J.M. Buhmann et al., Crowdsourcing the creation of image segmentation algorithms for connectomics. Front. Neuroanat. 9, 142 (2015)
6. L. Bao, Y. Song, Q. Yang, H. Yuan, G. Wang, Tree filtering: Efficient structure-preserving smoothing with a minimum spanning tree. IEEE TIP 23(2), 555-569 (2014)

7. Y. Boykov, O. Veksler, Graph cuts in vision and graphics: theories and applications. in Handbook of Mathematical Models in Computer Vision, ed. by N. Paragios, Y. Chen, O. Faugeras (Springer, Boston, 2006). https:// doi.org/10.1007/0-387-28831-7_5

8. W. Casaca, A. Paiva, E. Gomez-Nieto, P. Joia, L.G. Nonato, Spectral image segmentation using image decomposition and inner product-based metric. J. Math. Imaging Vis. 45(3), 227-238 (2013)

9. L. Cerrone, A. Zeilmann, F.A .Hamprecht. End-to-end learned random walker for seeded image segmentation. In: Proceedings of the IEEE Conference on computer vision and pattern recognition, pp. 12559-12568 (2019). https://doi.org/10.1109/CVPR.2019.01284

10. A. Challa, S. Danda, B.S. Daya Sagar, L. Najman, An introduction to Gamma-convergence for spectral clustering. In: Discrete Geometry for Computer Imagery - 20th IAPR International Conference, DGCI 2017, Vienna, Austria, September 19-21, 2017, Proceedings, vol. 10502, pp. 185-196 (2017)

11. A. Challa, S. Danda, B.S. Daya Sagar, L. Najman, Power spectral clustering on hyperspectral data. In: 2017 IEEE International Geoscience and Remote Sensing Symposium, IGARSS 2017, Fort Worth, TX, USA, July 23-28, 2017, pp. 2195-2198 (2017). https://doi. org/10.1109/IGARSS.2017.8127423

12. A. Challa, S. Danda, B.S.D. Sagar, L. Najman, Power spectral clustering. J. Math. Imaging Vis. 62(9), 11951213 (2020)

13. K.C. Ciesielski, A.X. Falcão, P.A.V. Miranda, Pathvalue functions for which Dijkstra's algorithm returns optimal mapping. J. Math. Imaging Vis. 60(7), 10251036 (2018)

14. M. Cordts, M. Omran, S. Ramos, T. Rehfeld, M. Enzweiler, R. Benenson, U. Franke, S. Roth, B. Schiele, The cityscapes dataset for semantic urban scene understanding. In: Proceedings of the IEEE Conference on computer vision and pattern recognition, pp. 3213-3223 (2016). https://doi.org/10.1109/CVPR.2016.350

15. T.H. Cormen, C.E. Leiserson, R.L. Rivest, C. Stein, Introduction to Algorithms (MIT Press, Cambridge, 2009)

16. C. Couprie, X. Bresson, L. Najman, H. Talbot, L. Grady, Surface reconstruction using power watershed. In: International Symposium on mathematical morphology and its applications to signal and image processing, vol. 6671 (Springer, 2011), pp. 381-392

17. C. Couprie, L. Grady, L. Najman, H. Talbot, Anisotropic diffusion using power watersheds. In: 2010 IEEE International Conference on image processing, 
pp. 4153-4156. IEEE, (2010). https://doi.org/10.1109/ ICIP.2010.5653896

18. C. Couprie, L. Grady, L. Najman, H. Talbot, Power watershed: a unifying graph-based optimization framework. IEEE Trans. Pattern Anal. Mach. Intell. 33(7), 1384-1399 (2011)

19. J. Cousty, G. Bertrand, L. Najman, M. Couprie, Watershed cuts: minimum spanning forests and the drop of water principle. IEEE Trans. Pattern Anal. Mach. Intell. 31(8), 1362-1374 (2009)

20. CREMI. Miccai challenge on circuit reconstruction from electron microscopy images. (2017). https://cremi.org/

21. S. Danda, A. Challa, B.S. Daya Sagar, L. Najman, Power tree filter: a theoretical framework linking shortest path filters and minimum spanning tree filters. In: Mathematical Morphology and Its Applications to Signal and Image Processing - 13th International Symposium, ISMM 2017, Fontainebleau, France, May 15-17, 2017, Proceedings, vol. 10225, pp. 199-210 (2017)

22. S. Danda, A. Challa, B.S.D. Sagar, L. Najman, Revisiting the isoperimetric graph partitioning problem. IEEE Access 7, 50636-50649 (2019)

23. S. Danda, A. Challa, B.S.D. Sagar, L. Najman, Some theoretical links between shortest path filters and minimum spanning tree filters. J. Math Imaging Vis. 61(6), 745-762 (2019)

24. A.X. Falcao, J. Stolfi, R. de Alencar Lotufo, The image foresting transform: theory, algorithms, and applications. IEEE PAMI 26(1), 19 (2004)

25. R.W. Floyd, Algorithm 97: shortest path. Commun. ACM 5(6), 345 (1962)

26. L. Grady, Fast, quality, segmentation of large volumesisoperimetric distance trees. In: Computer visionECCV 2006, 9th European Conference on Computer Vision, Graz, Austria, May 7-13, 2006, Proceedings, Part III, vol. 3953, pp. 449-462 (2006)

27. L. Grady, Random walks for image segmentation. IEEE PAMI 28(11), 1768-1783 (2006)

28. L. Grady, E.L. Schwartz, Isoperimetric graph partitioning for image segmentation. IEEE Trans. Pattern Anal. Mach. Intell. 28(3), 469-475 (2006)

29. L. Grady, E.L. Schwartz, Isoperimetric partitioning: a new algorithm for graph partitioning. SIAM J. Sci. Comput. 27(6), 1844-1866 (2006)

30. J. Grazzini, P. Soille, Edge-preserving smoothing using a similarity measure in adaptive geodesic neighbourhoods. Pattern Recognit. 42(10), 2306-2316 (2009)

31. C. Haigh, N. Chamba, A. Venhola, R. Peletier, L. Doorenbos, M. Watkins, M.H.F. Wilkinson, Optimising and comparing source extraction tools using objective segmentation quality criteria. arXiv preprint arXiv:2009.07586, (2020)

32. T. Hastie, R. Tibshirani, J. Friedman, The Elements of Statistical Learning: Data Mining, Inference, and Prediction (Springer, New York, 2009)

33. http://scikit-learn.org/stable/modules/generated/ sklearn.datasets.makeblobs.html.scikit-learndatasets. Accessed 12 Dec 2017

34. C. Jaquet, E. Andó, G. Viggiani, H. Talbot, Estimation of separating planes between touching $3 \mathrm{~d}$ objects using power watershed. In: International Symposium on mathematical morphology and its applications to signal and image processing, (Springer, 2013), pp. 452-463
35. R. Lerallut, É. Decencière, F. Meyer, Image filtering using morphological amoebas. Image Vis. Comput. 25(4), 395-404 (2007)

36. B. Mohar, Isoperimetric numbers of graphs. J. Comb. Theory Ser. B 47(3), 274-291 (1989)

37. J.-M. Morel, S. Solimini, Variational Methods in Image Segmentation: With Seven Image Processing Experiments, vol. 14 (Birkhäuser, Basel, 2012)

38. J.M. Musser, K.J. Schippers, M. Nickel, G. Mizzon, A.B. Kohn, C. Pape, J.U. Hammel, F. Wolf, C. Liang, A. Hernández-Plaza et al., Profiling cellular diversity in sponges informs animal cell type and nervous system evolution. BioRxiv, p. 758276, (2019)

39. L. Najman, Extending the PowerWatershed framework thanks to $\Gamma$-convergence. SIAM J. Imag. Sci. 10(4), 2275-2292 (2017)

40. T.X. Nguyen, G. Chierchia, L. Najman, A. Venhola, C. Haigh, R. Peletier, M.H.F .Wilkinson, H. Talbot, B. Perret, Cgo: multiband astronomical source detection with component-graphs. In: 2020 IEEE International Conference on image processing (ICIP). https://doi.org/10. 1109/ICIP40778.2020.9191276

41. J. Pont-Tuset, P. Arbelaez, J.T. Barron, F. Marques, J. Malik, Multiscale combinatorial grouping for image segmentation and object proposal generation. IEEE Trans. Pattern Anal. Mach. Intell. 39(1), 128-140 (2016)

42. J. Pont-Tuset, F. Marqués, Supervised evaluation of image segmentation and object proposal techniques. IEEE Trans. Pattern Anal. Mach. Intell. 38(7), 14651478 (2016)

43. A.S.G. Robotham, L.J.M. Davies, S.P. Driver, S. Koushan, D.S. Taranu, S. Casura, J. Liske, Profound: source extraction and application to modern survey data. Mon. Not. R. Astron. Soc. 476(3), 3137-3159 (2018)

44. C. Rother, V. Kolmogorov, A. Blake, "grabcut" interactive foreground extraction using iterated graph cuts. ACM Trans. Graph. (TOG) 23(3), 309-314 (2004)

45. J. Serra, Mathematical Morphology, vol. i (Academic, London, 1982)

46. J. Serra et al., Mathematical morphology for Boolean lattices. in Image Analysis and Mathematical Morphology, II: Theoretical Advances, (London Academic Press, 1988), pp. $37-58$

47. J.L. Sérsic, Observatorio Astronomico (Cordoba, Argentina, 1968)

48. J. Shi, J. Malik. Normalized cuts and image segmentation. In: Proceedings of IEEE Computer Society Conference on computer vision and pattern recognition, pp. 731-737. IEEE, (1997)

49. R. Szeliski, Computer Vision: Algorithms and Applications (Springer, London, 2010)

50. C. Tomasi, R. Manduchi, Bilateral filtering for gray and color images. In: Sixth International Conference on computer vision, 1998. ICCV 1998, pp. 839-846. IEEE, (1998)

51. V.V. Vazirani, Approximation Algorithms (Springer, Berlin, 2001)

52. A. Venhola. Evolution of dwarf galaxies in the fornax cluster. PhD Thesis, http://hdl.handle.net/11370/ 1bcc02c2-2c78-4cff-b801-147c31b000a8, (2019)

53. U. Von Luxburg, A tutorial on spectral clustering. Stat. Comput. 17(4), 395-416 (2007) 
54. S. Wolf, A. Bailoni, C. Pape, N. Rahaman, A. Kreshuk, U. Köthe, F.A. Hamprecht, The mutex watershed and its objective: Efficient, parameter-free graph partitioning. IEEE Trans. Pattern Anal. Mach. Intell. (2020). https://doi.org/10.1109/TPAMI.2020.2980827

55. S. Wolf, Y. Li, C. Pape, A. Bailoni, A. Kreshuk, F.A. Hamprecht, The semantic mutex watershed for efficient bottom-up semantic instance segmentation. In: European Conference on computer vision, vol. 12351 (Springer, 2020), pp. 208-224

56. S. Wolf, C. Pape, A. Bailoni, N. Rahaman, A. Kreshuk, U. Köthe, F.A. Hamprecht, The mutex watershed: Efficient, parameter-free image partitioning. in eds. V. Ferrari, M. Hebert, C. Sminchisescu, and Y. Weiss, Computer Vision-ECCV 2018 - 15th European Conference, Munich, Germany, September 8-14, 2018, Proceedings, Part IV, volume 11208 of Lecture Notes in Computer Science, (Springer, 2018), pp. 571-587
57. X. Lijuan, F. Wang, L. Dempere-Marco, Q. Wang, Y. Yang, H. Xiaopeng, Path-based analysis for structurepreserving ima ge filtering. J. Math. Imaging Vis. 62(2), 253-271 (2020)

58. Q. Yang, Stereo matching using tree filtering. IEEE PAMI 37(4), 834-846 (2015) 